\title{
Optimization of Linear Multielement Antennas for Selection Combining by Means of a Butler Matrix in Different MIMO Environments
}

\author{
Alfred Grau, Jordi Romeu, Sebastián Blanch, Lluis Jofre, Member, IEEE, and Franco De Flaviis
}

\begin{abstract}
An optimized linear multielement antenna (MEA) is presented for selection combining schemes that improves the selection diversity gain and selection diversity capacity in medium and low multipath environments, with respect to the performance achieved with a simple uniform linear array (ULA) using omnidirectional antennas, while it performs equally as well as a ULA in highly scattered environments. An analytical investigation based on the analysis of the correlation coefficients, together with simulations and extensive measurements, have been carried out for different fading multiple-input multiple-output environments ranging from line of sight (LOS) to non-LOS. Two MEAs are compared: a simple ULA with omnidirectional antennas and a MEA combining a ULA and a Butler matrix. The measurement results show that the nature of the proposed MEA is such that it is adaptive to any propagation scenario by simultaneously taking advantage of beamforming gain and signal diversity gain.
\end{abstract}

Index Terms-Beamforming gain, Butler matrix, channel capacity, diversity gain, linear multielement antenna, multiple-input multiple-output (MIMO), selection combining.

\section{INTRODUCTION}

I $\mathrm{N}$ multiple-input multiple-output (MIMO) [1] wireless systems, multielement antennas (MEA) such as uniform linear arrays (ULAs or linear MEA) can be used to increase the transmission rate and improve the reliability of the transmitted signals when they are used in combination with spatial multiplexing techniques and space-time codes, respectively. Channel state information (CSI) is normally assumed at the receiver. If CSI is also available at the transmitter, beamforming can be used to further improve the performance of a MIMO system by focusing the energy of the transmitted codeword toward some desired direction in space. One technique or another is chosen for optimal performance depending on the availability and the quality of CSI at the transmitter and at the receiver, the

Manuscript received September 16, 2005; revised May 10, 2006. This work was supported in part by Balsells Fellowships, the California-Catalonia Engineering Innovation Programs 2004/2005, Broadcom Corporation under MICRO Grant 04-025, and the National Science Foundation under Award ECS-0424454. The work of J. Romeu was supported by The Ministerio Educación y Ciencia of Spain under Grant TEC-2004-04866-C04-02.

A. Grau and F. De Flaviis are with the Electrical Engineering and Computer Science Department, University of California at Irvine, Irvine, CA 92697 USA (e-mail: agrau@uci.edu; franco@uci.edu).

J. Romeu, S. Blanch, and L. Jofre are with the Department of Signal Theory and Communications, Technical University of Catalonia, Barcelona 08034, Spain (e-mail: romeu@tsc.upc.edu).

Color versions of Figs. 1, 2, and 14 are available online at http://ieeexplore. ieee.org.

Digital Object Identifier 10.1109/TAP.2006.883971 scattering characteristics of the channel, the characteristics of the MEAs, and the signal-to-noise ratio (SNR) regime.

In [2], it is shown that if perfect CSI is available at both the transmitter and the receiver, the channel capacity $(C)$ can be attained by spatial water-filling on the eigenmodes of the channel matrix and maximum ratio combining (MRC) at the receiver. If CSI is not available at the transmitter, then uniform power loading of the transmitting antennas and MRC at the receiver gives the optimal performance.

At the receiver front end, MRC is normally used in the combiner because it is well known that MRC is the optimum theoretical diversity combining method for branch signals having an arbitrary signal amplitude fading distribution [3]. Therefore, in propagation channels with either independent or correlated channel fading, and Rayleigh or Rician envelope distribution, a receiver using MRC performs optimally. Despite all this, one drawback of MRC is its cost of implementation because one radio-frequency ( $\mathrm{RF}$ ) chain is needed for each receiving antenna. In fact, this is one of the major drawbacks of MIMO, because RF components are expensive and their integration is difficult. MRC coherently combines all the received signals, and to do this it requires perfect CSI. This brings additional overhead in the transmission signaling techniques by means of training periods and requires the estimation of the channel coefficients, which also increases the complexity of the receiver. Alternatively, if some spectral efficiency can be sacrificed, selection combining (SC) arises as a most cost-effective combining technique because only uses one RF chain. SC chooses the path with the highest SNR and performs detection based on the signal from the selected path. It uses limited CSI at the receiver based on the instantaneous SNR of each antenna and hence does not require to estimate the channel coefficients. Because SC is a suboptimal combining scheme compared to MRC, the diversity gain using $\mathrm{SC}$ is in general lower than that achieved using MRC. However, SC can still provide very competitive diversity gains at the expense of very low complexity and cost, especially in rich scattering environments with independent fading and Rayleigh envelope distribution [4]

On the other hand, most practically occurring cellular channels exhibit fading correlation and Rician envelope distribution, due to a nonuniform power azimuth spectrum (PAS) at the base station. Nonuniform PAS can arise in propagation environments with poor scattering and when there exists a dominant line-of-sight (LOS) propagation component. In such channels, the performance of SC deteriorates much faster than that of full complexity MRC schemes [5]. 


\section{A. Proposed Optimal Linear MEA for Selection Combining}

To compensate for the detrimental effect of correlated and Rician channels on SC schemes, in this paper we propose an optimized linear MEA that combines a ULA and a Butler matrix to arrange omnidirectional antenna patterns into directive beams. For the seek of clarifying, an ideal Butler matrix is a passive, reciprocal, and lossless network with $n_{R}$ input and $n_{R}$ output ports, such that a signal introduced at one of the inputs produces equal amplitude excitations at all the output ports but with a constant phase difference among them, resulting in radiation at a certain angle in space when combined with a ULA. A signal introduced at another input port results in radiation towards a different angle in space. A Butler matrix in fact performs a spatial fast Fourier transform, and up to $n_{R}$ orthogonal directive beams can be generated when combined with a ULA.

In particular, the proposed MEA is built by connecting the output ports of a Butler matrix to the input ports of a ULA, such that the input ports for the new MEA are the input ports of the Butler matrix. A detailed description is given in Section III. As will be shown in Sections IV-VI, when compared to a simple ULA, the proposed MEA configuration improves the performance of SC by exploiting the benefits of beamforming in low scattered environments, the benefits of spatial diversity in rich scattered environments, or simultaneously taking advantage of both techniques in intermediate propagation conditions. Similarly as in [6], the architecture can adapt to different propagation conditions, ranging from line-of-sight (LOS) to non-LOS (NLOS), but in our case, the beamforming capability is done by using an external circuitry and not via digital signal processing. Because the beamforming is done via hardware before the combiner, only one RF chain is needed, hence reducing the cost of implementation and complexity. In the proposed setting, the claimed performance gains are achieved before the front-end and therefore before the detector. Interestingly, also notice that despite the fact that the proposed architecture performs well in different environments and is able to adapt itself, the Butler matrix network is static and does not dynamically adapt any of its hardware component. This is one of the advantages of multidirective-beam MEAs.

The proposed linear MEA is optimal in two ways. First, assuming that there are not electronically adjustable phase shifts components on the external circuitry, the Butler matrix is the only static microwave network that will not degrade the performance of a ULA in rich scattered environments. This will be further explained in Section IV-A3 using [7] and [8]. Secondly, because the Butler matrix is a unique lossless reciprocal microwave network with which the full array gain of a ULA can realized in each beam [7], [8], the proposed linear MEA is a multidirective-beam MEA with which similar beamforming gains can be achieved in any direction of the azimuth plane. For the seek of clarifying, through this paper, we will also refer to a ULA as a multiomnidirectional-beam MEA.

Notice that the proposed architecture can be used at the transmitter front-end as well, although its study is left as future work.

Recently, it has been brought to our attention that this configuration has been considered in [9]. In our approach, we include a performance analysis based on the correlation coefficients and validate the benefits of the optimized MEA through simulations and extensive measurements over different propagation conditions ranging from LOS to NLOS.

\section{B. Organization}

To investigate the benefits of the proposed MEA configuration over a simple ULA, those two MEA configurations have been fabricated and compared. In Section II, the system model and the two figures of merit, the selection diversity gain and the selection diversity capacity, are presented. In Section III, the two compared MEA configurations are described. In Section IV, their performance in terms of the two figures of merit is predicted based on the analysis of its correlation coefficients in different propagation scenarios. In Section V, simulation results are shown, and in Section VI, they are verified through measurements.

\section{System MODEL AND FiguRES OF MERIT}

\section{A. System Model}

Consider a single-input multiple-output (SIMO) system, consisting of a receiving diversity combining scheme with four receiving antennas $\left(n_{R}=4\right)$ and one transmitting antenna $\left(n_{T}=\right.$ 1). We assume that the channel fading at each receiving branch is frequency nonselective and changes slowly with equally correlated Rician distributed envelop statistics. The received signal at the $k$ th diversity branch can be expressed as

$$
r_{k}=h_{k} s+n_{k}
$$

where $h_{k}=\alpha_{k} e^{j \phi_{k}}$ is the channel gain associated with the $k$ th branch, where $\left\{k \mid 1 \leq k \leq n_{R}\right\}, s$ is the transmitted signal with energy per symbol $E s$ and $n_{k}$ is additive white Gaussian noise (AWGN) with identical power spectral density $N_{0} / 2$ per dimension.

If an external circuitry with transfer function $\mathbf{W}$ is inserted between the combiner and the receiving antennas, the received signal at the $k$ th diversity branch can be expressed as

$$
r_{k}=\sum_{i=1}^{n_{R}} w_{k i} h_{i} s+\sum_{i=1}^{n_{R}} w_{k i} n_{i}=\hbar_{k} s+\eta_{k}
$$

where $\hbar_{k}=\beta_{k} e^{j \theta_{k}}$ and $\mathbf{W}$ is a matrix with dimensions $n_{R} \times n_{R}$ and entries $w_{k i}$. If the external circuitry corresponds to a Butler matrix, the entries of $\mathbf{W}$ are given by $w_{k i}=1 / \sqrt{n_{R}}\left(e^{j\left(2 \pi(i-1) / n_{R}\right)}\right)^{k-1}\left(e^{j(3 \pi) / 4}\right)^{k-1}[10]$, where $\left\{k, i \mid 1 \leq k, i \leq n_{R}\right\}, j=\sqrt{-1}$, and $k$ indexes the input ports of the Butler matrix and $i$ the output ports of the Butler matrix connecting to the antennas of the ULA. In that case, since each row and column of $\mathbf{W}$ has length one, after the combiner, the noise power with or without the Butler matrix remains the same, $E\left[\left|n_{k}\right|^{2}\right]=E\left[\left|\eta_{k}\right|^{2}\right]$.

\section{B. Figures of Merit}

In this paper, we use two figures of merit to evaluate the performance of the proposed architecture: the selection diversity gain and the selection diversity capacity. 
Selection combining is a diversity technique that chooses the path with the highest SNR at each time instant and performs detection based on the signal from the selected path. No CSI is assumed at the transmitter, so equal power distribution is assumed among transmit antennas. Partial CSI is assumed at the receiver, consisting on the SNR available at each receiving antenna at any time instant. Using the model in (1), the instantaneous SNR at the output of the combiner after SC can be expressed as

$$
\rho_{s c}=\rho \max \left\{\alpha_{1}^{2}, \ldots, \alpha_{n_{R}}^{2}\right\}
$$

where $\rho=E_{s} / N_{0}$ is the instantaneous SNR at each antenna. The selection combining diversity gain $G_{s c}$ can be defined as the increase on the average SNR due to the combined signal from the $n_{R}$ receiving antennas, with respect to the average SNR at a single antenna

$$
G_{s c}=E\left[\max \left\{\alpha_{1}^{2}, \ldots, \alpha_{n_{R}}^{2}\right\}\right]
$$

Selection diversity capacity is that offered by the best of the $n_{R}$ receiving channels [1], corresponding to the $n_{R}$ receiving antennas, and can be expressed as

$$
C_{s c}=\log _{2}\left(1+\rho \max _{k}\left|h_{k}\right|^{2}\right)
$$

where the maximization is over $\left\{k \mid 1 \leq k \leq n_{R}\right\}$. Therefore, the system selects the port that maximizes the instantaneous capacity at each time instant.

Notice that while the performance of selection diversity combining is inferior to optimum combining, it can be simpler to implement. For the seek of clarifying, throughout this paper, the selection diversity capacity is also compared with the optimum capacity, that is, the theoretical limit on MIMO capacity for a given propagation condition and SNR, based on equation

$$
C=\log _{2}\left(\operatorname{det}\left(\mathbf{I}_{\mathbf{R}}+\frac{\rho}{n_{T}} \mathbf{h h}^{H}\right)\right)
$$

where $(\cdot)^{H}$ indicates transpose conjugate, $\mathbf{I}_{\mathbf{R}}$ is an identity matrix with dimensions $n_{R} \times n_{R}$, and $\mathbf{h}$ is the normalized channel matrix [11] with dimensions $n_{R} \times n_{T}$.

leqalignno\{

Equations (3)-(6) are also valid if a Butler matrix with transfer function $\mathbf{W}$ is inserted between the combiner and the receiving antennas by replacing the variables $\alpha_{k}, h_{k}, \mathbf{h}=\left(h_{1}, \ldots, h_{n_{R}}\right)^{T}$ by $\beta_{k}, \hbar_{k}, \mathbf{h}=\left(\hbar_{1}, \ldots, \hbar_{n_{R}}\right)^{T}$, respectively.

\section{DESCRIPTION OF THE TWO COMPARED MEA CONFIGURATIONS}

To investigate the benefits of the proposed MEA configuration over a simple ULA, the following two MEA configurations have been designed, fabricated, and compared.

1) Multiomnidirectional-Beam MEA: A ULA of four omnidirectional quarter-wave monopole antennas spaced $0.5 \lambda$,

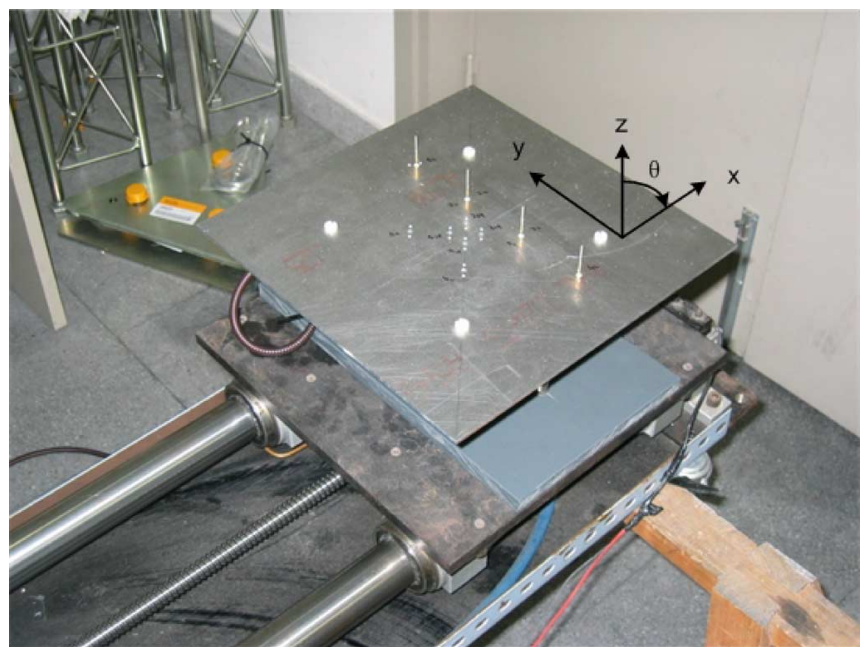

Fig. 1. Picture of the multiomnidirectional-beam MEA consisting of a ULA of omnidirectional monopoles over a finite ground plane, mounted on the automatized measurement setup.

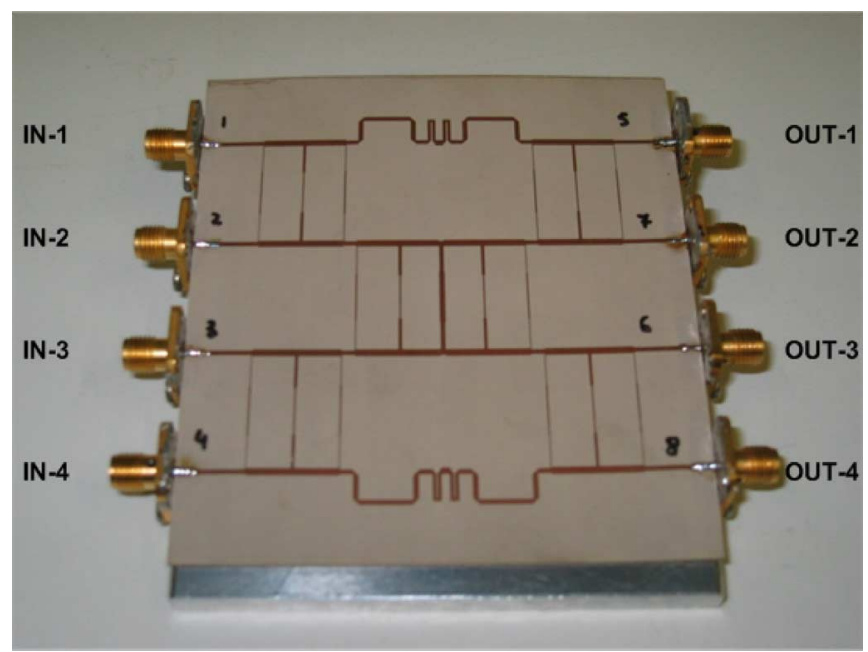

Fig. 2. Picture of the dual-band Butler matrix network used on in the multidirectional-beam MEA. The total area of the Butler matrix is $60 \times 60 \mathrm{~mm}$.

operating at $2.45 \mathrm{GHz}$, and over a finite ground plane of dimensions $30 \times 30 \mathrm{~cm}$. A picture of it can be seen in Fig. 1 . Throughout this paper, this configuration may be referred to as MEA 1.

2) Multidirective-Beam MEA: The ULA in configuration 1 with its input ports connected to the output ports of the Butler matrix described in [10]. This MEA arranges the omnidirectional antennas on directive beams and corresponds to the optimized linear MEA for SC schemes proposed in Section I-A. The inputs ports for the new MEA are the input ports of the Butler matrix. Throughout this paper, this configuration may be referred to as MEA 2.

The Butler matrix in [10] is a $4 \times 4$ network, fabricated on RO3006 with relative dielectric constant 6.15 , thickness $0.635 \mathrm{~mm}$, and metallization thickness $0.017 \mathrm{~mm}$. Fig. 2 shows a picture of the used Butler matrix. 


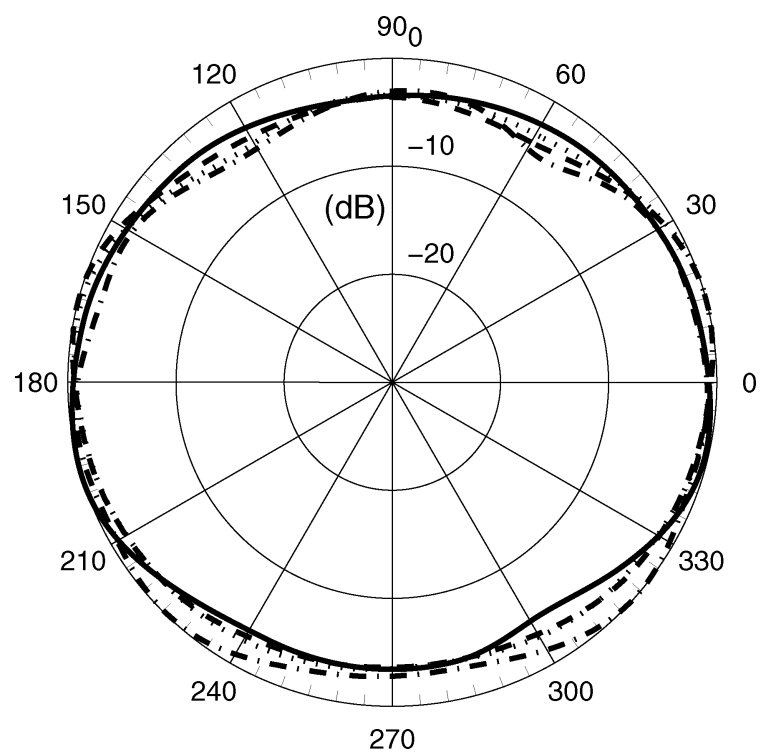

Fig. 3. Normalized radiation patterns (in $\mathrm{dB}$ ) versus $\phi$ angle in the $\theta=\pi / 2$ plane, for each of the input ports of the multiomnidirectional-beam linear MEA.

The tridimensional radiation patterns of the two prescribed MEA have been measured at the anechoic chamber of the Technical University of Catalonia, Spain. Fig. 3 shows the superposition of the copolar components of the normalized radiation patterns versus $\phi$ angle for the $\theta=\pi / 2$ cut, for each one of the four input ports of multiomnidirectional-beam MEA. In all the figures, the reference coordinate system is the one shown in Fig. 1. For the azimuth plane $(\theta=\pi / 2$ cut), the radiation patterns can be considered omnidirectional. The directivity was computed from the radiation patterns and was found to be in the range of 6.6 to $6.9 \mathrm{dBi}$, depending on the input port. The radiation patterns show little deformation and similar directivity values, as expected from monopole antennas spaced $d=0.5 \lambda$. Notice that the theoretical directivity of a quarter-wavelength monopole over an infinite ground plane is $5.15 \mathrm{dBi}$ [12], which is slightly smaller than the measured values. Those differences can be explained by the fact that the measured monopoles are not isolated and the ground plane is finite.

Fig. 4 shows the normalized radiation patterns versus $\phi$ angle for the $\theta=\pi / 2$ cut, for each one of the four input ports of multidirectional-beam MEA. In this case, the directivity at ports 1 and 4 is approximately $9.7 \mathrm{dBi}$, while at ports 2 and 3 it is around $11.5 \mathrm{dBi}$.

\section{Performance Analysis}

In [13] and [14], it is shown that the selection diversity gain, the selection diversity capacity, and the optimum capacity depend on the values of the correlation coefficients seen from the accessible ports of the MEAs at the receiver and transmitter end. In fact, the channel matrix $\mathbf{h}$ can be expanded into $\mathbf{h}=$ $\mathrm{C}_{R}^{1 / 2} \mathrm{gC}_{T}^{1 / 2}$, where $\mathrm{C}_{R}$ is the correlation matrix at the receiver with dimensions $n_{R} \times n_{R}, \mathrm{C}_{T}$ is the correlation matrix at the transmitter with dimensions $n_{T} \times n_{T}$, and $\mathrm{g}$ is a $n_{R} \times n_{T}$ matrix that represents the path gains channel coefficients, without including the correlation effects. Because $n_{T}=1$, we assume $\mathrm{C}_{T}=1$ and therefore $\mathbf{h}=\mathrm{C}_{R}^{1 / 2} \mathrm{~g}$. On the other hand, (5) and

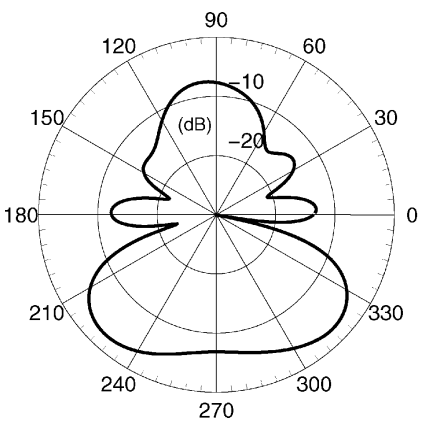

(a)

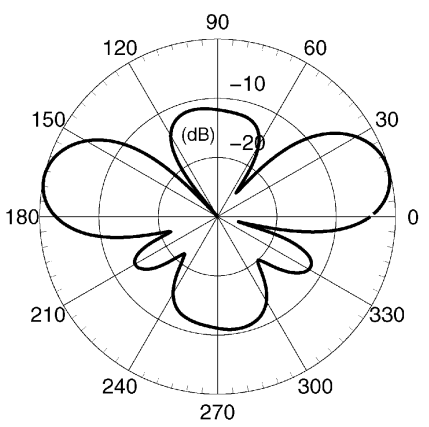

(c)

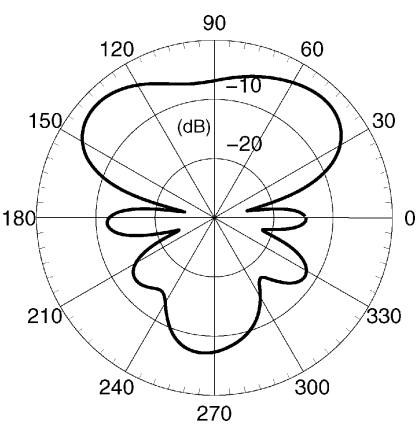

(b)

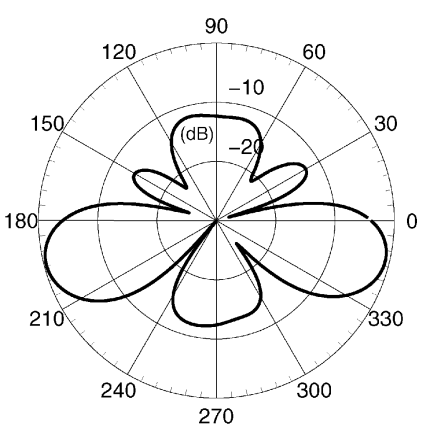

(d)
Fig. 4. Normalized radiation patterns (in $\mathrm{dB}$ ) versus $\phi$ angle in the $\theta=\pi / 2$ plane, for each of the input ports of the multidirectional-beam linear MEA. (a) Port 1, (b) Port 2, (c) Port 3, and (d) Port 4.

(6) can be rewritten including explicitly the effects of the correlation as follows

$$
\begin{aligned}
C_{s c} & =\log _{2}\left(1+\rho \max _{k}\left|\left(\mathrm{C}_{\mathbf{R}}^{\mathbf{1} / \mathbf{2}} \mathbf{g}\right)_{k}\right|^{2}\right) \\
C & =\log _{2}\left(\operatorname{det}\left(\mathbf{I}_{\mathbf{R}}+\frac{\rho}{n_{T}} \mathbf{C}_{\mathbf{R}} \mathbf{g g}^{\mathbf{H}}\right)\right) .
\end{aligned}
$$

In the next sections, we will estimate the correlation matrix at the receiver $\mathrm{C}_{R}$ to predict the differences in the selection diversity gain and selection diversity capacity, among the two prescribed MEA, in different propagation scenarios. Notice, however, that from the correlation coefficient alone, one can not estimate the absolute performance of each MEA.

To compute the coefficients of the correlation matrix $\mathrm{C}_{R}$, one can follow the approach in [15] and use the formula

$$
\mathrm{C}_{\mathbf{R}_{\mathbf{i j}}}=\sqrt{D_{i} D_{j}} \iint_{4 \pi} \mathbf{F}_{\mathbf{i}}(\boldsymbol{\Omega}) \mathbf{F}_{\mathbf{j}}(\boldsymbol{\Omega})^{H} P(\Omega) d \Omega
$$

where $\mathbf{F}_{\mathbf{i}}(\boldsymbol{\Omega})$ and $D_{i}$ are the normalized radiation pattern and the maximum directivity associated to the $i$ th accessible port of the MEA, respectively, $P(\Omega)$ represents the PAS over the $4 \pi$ sphere, $\Omega=(\theta, \phi)$, and $0 \leq \mathrm{C}_{\mathbf{R}_{\mathbf{i j}}} \leq 1$. It is clear that the entries of the correlation matrix can be computed from the radiation patterns associated to the ports of the MEA and the PAS. Therefore, by inspection of (7), and from [13], it is also clear that the achievable selection diversity gain and selection diversity capacity depends ultimately on the shape of the radiation pattern associated to the ports of the MEAs and the distribution of the PAS, which is fixed by the characteristics of the scattering channel response. 


\section{A. Performance Analysis in an Ideal NLOS Scenario}

1) Estimation of the Correlation Matrix $\mathrm{C}_{\mathbf{R}}$ : In rich scattered environments, in order not to lower the rank of the channel matrix $\mathbf{h}$, in general it is desirable to have $\mathbf{C}_{\mathbf{R}}=\mathbf{I}_{\mathbf{R}}$. This means that the MEA has its accessible ports decorrelated, and therefore the achievable diversity gain and capacity depend uniquely on the scattering channel response. In other words, the receiving MEA does not impact negatively on the system.

Fortunately, under the assumption of uniform PAS $P(\Omega)=$ $1 / 4 \pi$ as in the case of a perfectly rich scattered environment (ideal NLOS), the coefficients in (9) can also be computed from the measured scattering parameters among the accessible ports of the MEA [15] using

$$
\mathrm{C}_{\mathbf{R}}=\mathbf{I}_{\mathbf{R}}-\mathbf{S}_{\mathbf{A}}{ }^{H} \mathbf{S}_{\mathbf{A}}
$$

where $\mathbf{S}_{\mathbf{A}}$ is the scattering matrix of the MEA. For the seek of clarifying, a scattering matrix is a matrix description of a microwave network as seen from its accessible ports that relates the voltage waves incident on the ports to those reflected from the ports [16].

The condition $\mathbf{S}_{\mathbf{A}}=\mathbf{0}$ is therefore desirable because in that case $\mathrm{C}_{\mathbf{R}}=\mathbf{I}_{\mathbf{R}}$. The physical meaning of this condition from a transmission point of view can be seen as follows: if $\mathbf{a}$ is the excitation vector for any given MEA, the power dissipated on the antennas $P_{D}$ is given by

$$
P_{D}=\mathbf{a}^{H}\left(\mathbf{I}_{\mathbf{R}}-\mathbf{S}_{\mathbf{A}}{ }^{H} \mathbf{S}_{\mathbf{A}}\right) \mathbf{a} .
$$

From the condition $\mathbf{S}_{\mathbf{A}}=\mathbf{0} \Rightarrow \mathbf{S}_{\mathbf{A}}{ }^{H} \mathbf{S}_{\mathbf{A}}=\mathbf{0}$, we have $P_{D}=\mathbf{a}^{H} \mathbf{a}=|\mathbf{a}|^{2}$, and all the available power is radiated by the antennas in the lossless case. By virtue of the reciprocity theorem, the received power is totally delivered into the loads, when the above condition is satisfied. Interestingly, this gives us a more physical insight on the meaning of the decorrelation conditions of having $\mathbf{S}_{\mathbf{A}}=\mathbf{0}$, in the sense that this is equivalent to maximizing the power transfer from the receiving antennas to the loads.

In addition, for the ideal NLOS case, equating (9) and (10), we see that the condition $\mathbf{S}_{\mathbf{A}}=\mathbf{0}$ is equivalent to having orthogonal radiation patterns among the ports of the MEA, that is, $\left\langle\mathbf{F}_{\mathbf{i}}(\boldsymbol{\Omega}), \mathbf{F}_{\mathbf{j}}(\boldsymbol{\Omega})\right\rangle=0$ and $\left\langle\mathbf{F}_{\mathbf{i}}(\boldsymbol{\Omega}), \mathbf{F}_{\mathbf{i}}(\boldsymbol{\Omega})\right\rangle=1$, where $\langle\cdot, \cdot\rangle$ represents the inner product.

Next, we estimate the entries of the correlation matrix $C_{\mathbf{R}}$ for the two prescribed MEAs in an NLOS environment, from the measured scattering parameters $\mathbf{S}_{\mathbf{A}}$ and from its measured radiation patterns.

2) Performance Prediction of the Two Prescribed MEA Configurations: Figs. 5 and 6 show the measured correlation coefficients according to (10) for the multiomnidirectional-beam and multidirectional-beam MEAs, respectively. One can observe that the difference gap among the self-correlation and cross-correlation curves is approximately 13 and $15 \mathrm{~dB}$ for the multiomnidirectional-beam MEA and multidirectional-beam MEA, respectively, at a central frequency of $2.45 \mathrm{GHz}$. In addition, the self-correlation coefficients are approximately $0 \mathrm{~dB}$ in all cases. This indicates that there is no power loss due to mismatch and the mutual coupling between the antennas is

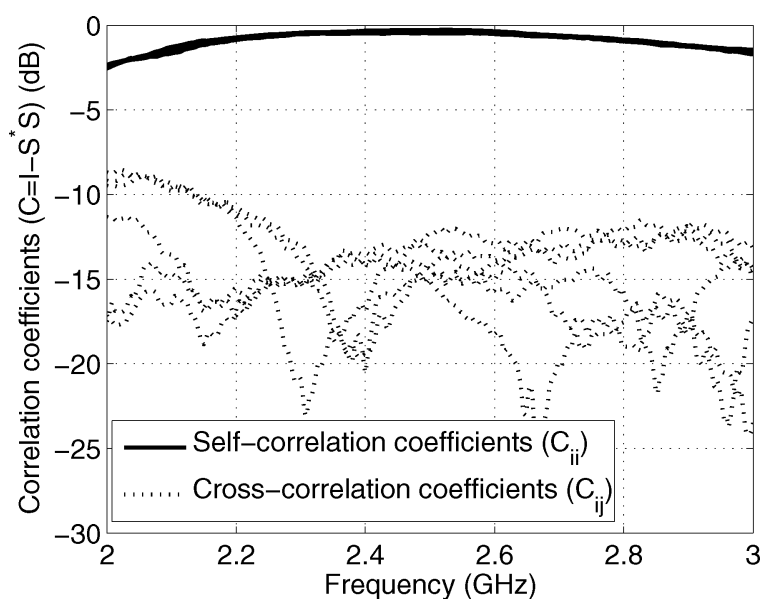

Fig. 5. Correlation coefficients of the multiomnidirectional-beam MEA (MEA 1) versus frequency.

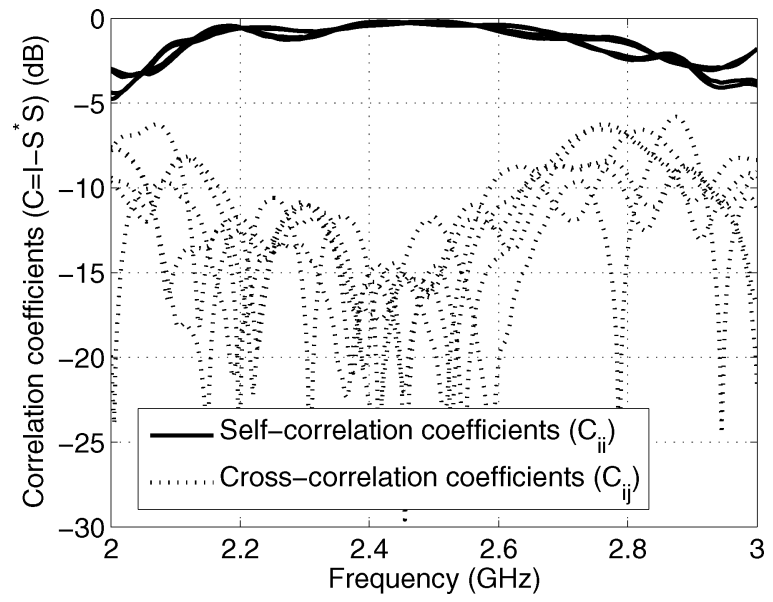

Fig. 6. Correlation coefficients of the the multidirectional-beam MEA (MEA 2) versus frequency.

negligible; therefore the ports of the two prescribed MEA are decorrelated in a NLOS environment. That is, $\mathrm{C}_{\mathbf{R}} \approx \mathbf{I}_{\mathbf{R}}$ is verified.

Equivalently, this can also be seen from Figs. 7 and 8, which show the measured scattering parameters for the multiomnidirectional-beam and multidirectional-beam MEAs, respectively. All the scattering parameters are small enough, below $-13 \mathrm{~dB}$ at $2.45 \mathrm{GHz}$, to consider that the condition $\mathbf{S}_{\mathbf{A}} \approx 0$ is satisfied. The input ports of the two prescribed MEA are simultaneously matched and decoupled.

Although not shown, the correlation entries were also calculated using (9) and from the measured radiation patterns shown in Figs. 3 and 4. Interestingly, the values agreed well with those obtained from the scattering parameters. The orthogonality conditions $\left\langle\mathbf{F}_{\mathbf{i}}(\boldsymbol{\Omega}), \mathbf{F}_{\mathbf{j}}(\boldsymbol{\Omega})\right\rangle \approx 0,\left\langle\mathbf{F}_{\mathbf{i}}(\boldsymbol{\Omega}), \mathbf{F}_{\mathbf{i}}(\boldsymbol{\Omega})\right\rangle \approx 1$ were verified for the two prescribed MEAs.

Summarizing, under the assumption of uniform PAS, the two prescribed MEAs equally well satisfy the decorrelation condition of the input ports, or equivalently, the orthogonal condition of the radiation patterns. Due to the fact that when $\mathbf{C}_{\mathbf{R}}=\mathbf{I}_{\mathbf{R}}$ the performance of a MIMO system does not depend on the characteristics of the MEA but only on the scattering response of 


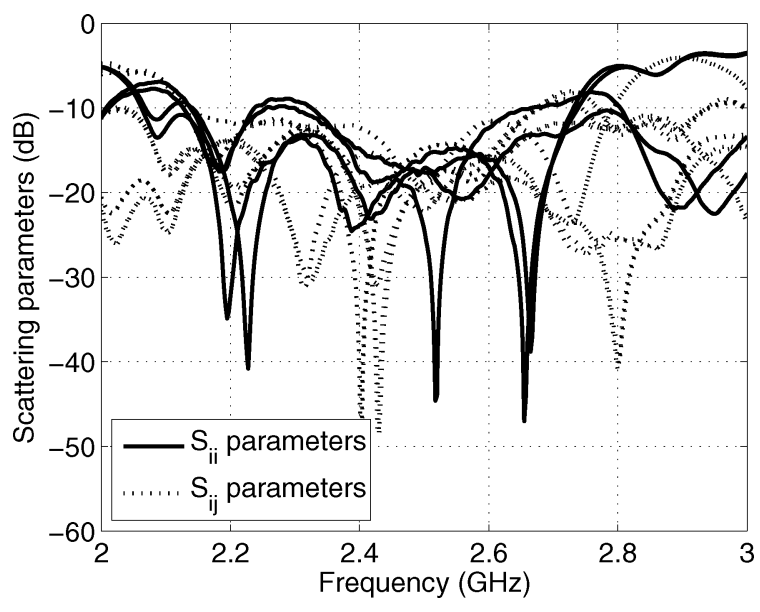

Fig. 7. Scattering parameters of the multiomnidirectional-beam MEA (MEA 1) versus frequency.

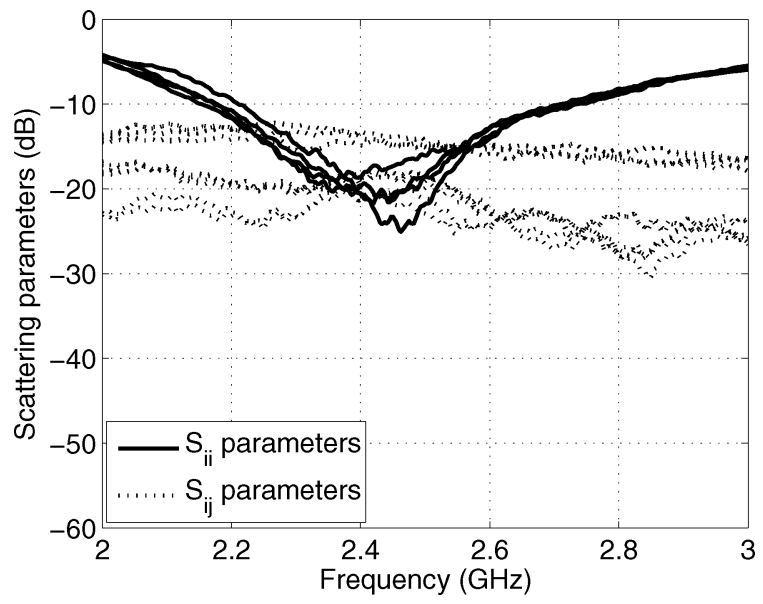

Fig. 8. Scattering parameters of the the multidirectional-beam MEA (MEA 2) versus frequency.

the channel, we can predict that the two prescribed MEAs will perform equally well in terms of selection diversity gain and selection diversity capacity in an NLOS scenario.

3) Other Considerations: The shape of the radiation patterns is seen to be irrelevant in an NLOS scenario as long as the total captured radiation energy is the same for the two prescribed MEAs, and the orthogonality condition of the radiation patterns is satisfied. For the multiomnidirectional-beam MEA, the pattern orthogonality condition is satisfied because the omnidirectional antennas are spaced $d=0.5 \lambda$. For the multidirective-beam MEA, the condition is satisfied because each radiation pattern is pointing to a different location in space.

On the other hand, as discussed in Section I-A and from the above results, the Butler matrix is the only static microwave network that will not degrade the performance of a ULA in rich scattered environments. This is because the Butler matrix is the only network providing orthogonal radiation patterns of similar gain in a ULA with omnidirectional antennas spaced $d=0.5 \lambda$, and under the assumption of uniform PAS [7], [8].

Finally, from Figs. 7 and 8, notice that the bandwidth, defined at $-10 \mathrm{~dB}$, for the multiomnidirectional-beam MEA and multidirective-beam MEA are approximately equal, which shows that the Butler matrix does not significantly reduce the operational frequency range with respect to that of a ULA.

\section{B. Performance Analysis in an Ideal LOS Scenario}

1) Estimation of the Correlation Matrix $\mathrm{C}_{\mathbf{R}}$ : If, for a given propagation channel, the assumption of uniform PAS does not hold, then the correlation matrix at the receiver has to be computed using (9). For the seek of simplicity, assume that the incoming signals are arriving within the azimuth plane. Equation (9) can be simplified to

$$
\mathrm{C}_{\mathbf{R}_{\mathbf{i} \mathbf{j}}}=\sqrt{D_{i} D_{j}} \int_{2 \pi} \mathbf{F}_{\mathbf{i}}(\phi) \mathbf{F}_{\mathbf{j}}(\phi)^{H} P(\phi) d \phi
$$

For convenience, let us first decompose the channel matrix into an LOS component $\mathbf{h}_{\mathrm{LOS}}$ and NLOS component $\mathbf{h}_{\mathrm{LOS}}$, as suggested in [17]. Recalling the fact that $n_{T}=1$, and including explicitly the correlation effects, we have $\mathbf{h}_{\mathrm{LOS}}=\mathrm{C}_{R_{\mathrm{LOS}}}^{1 / 2} \mathbf{g}_{\mathrm{LOS}}$, $\mathbf{h}_{\text {NLOS }}=\mathrm{C}_{R_{\mathrm{NLOS}}}^{1 / 2} \mathbf{g}_{\mathrm{NLOS}}$. Notice that $\mathbf{g}_{\mathrm{LOS}}$ and $\mathbf{g}_{\mathrm{NLOS}}$ are the path gains for the LOS and NLOS components, respectively, without including the correlation effects, and $\mathrm{C}_{R_{\mathrm{LOS}}}$ and $\mathrm{C}_{R_{\mathrm{NLOS}}}$ are the correlation matrices at the receiver for the LOS and NLOS components, respectively. In that case, we have

$$
\begin{aligned}
\mathbf{h} & =\mathrm{C}_{\mathbf{R}}{ }^{1 / 2} \mathbf{g} \\
& =\sqrt{\frac{K}{K+1}} \mathrm{C}_{R_{\mathrm{LOS}}}^{1 / 2} \mathbf{g}_{\mathrm{LOS}}+\sqrt{\frac{1}{K+1}} \mathrm{C}_{R_{\mathrm{NLO}}}^{1 / 2} \mathbf{g}_{\mathrm{NLOS}}
\end{aligned}
$$

where $\mathbf{g}_{\mathrm{LOS}}=1$. Now, for a signal arriving from direction $\phi_{R}$, and assuming omnidirectional radiation patterns for the receiving antennas, that is, $\mathbf{F}_{\mathbf{i}}\left(\phi_{R}\right)=1$, let us define the vector $\mathbf{a}\left(\phi_{R}\right)$ with dimensions $n_{R} \times 1$ as

$$
\mathbf{a}\left(\phi_{R}\right)=\left(1, e^{j 2 \pi\left(\sin \left(\phi_{R}\right)\right)}, \ldots, e^{\left.j 2 \pi\left(n_{R}-1\right) \sin \left(\phi_{R}\right)\right)}\right)^{T} .
$$

In an ideal LOS scenario, with $K \rightarrow \infty$, one can simplify the expression for the channel matrix given in (13) into $\mathbf{h}=$ $\mathrm{C}_{R_{\mathrm{LOS}}}^{1 / 2}$, where we notice that $\mathrm{C}_{R_{\mathrm{LOS}}}^{1 / 2}$ has dimensions $n_{R} \times 1$ and $\mathrm{C}_{R_{\mathrm{LOS}}}=\mathrm{C}_{R_{\mathrm{LOS}}}^{1 / 2}\left(\mathrm{C}_{R_{\mathrm{LOS}}}^{1 / 2}\right)^{H}$. As shown in [18] and using the above notation, for the case of the multiomnidirectional MEA we can write

$$
\mathrm{C}_{R_{\mathrm{LOS}}}^{1 / 2}=\mathbf{a}\left(\phi_{R}\right)
$$

and the selection diversity capacity (7) is given by

$$
C_{s c}=\log _{2}\left(1+\rho \max _{k}\left|\left(\mathbf{a}\left(\phi_{R}\right)\right)_{k}\right|^{2}\right) .
$$

From (16), it is easy to see that the maximum possible value that this expression can achieve is $\log _{2}(1+\rho)$, which is the capacity value for a single-input single-output (SISO) channel with omnidirectional antennas at both ends.

On the other hand, in the case of the multidirectional MEA, which combines a Butler matrix with transfer function $\mathbf{W}$, as 


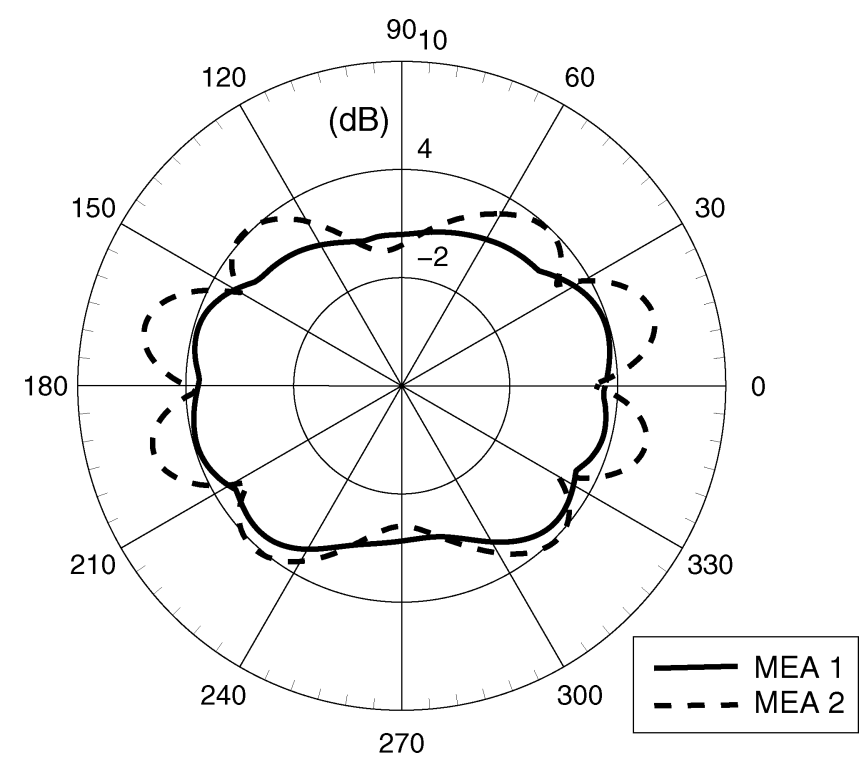

Fig. 9. Resultant directivity pattern (in $\mathrm{dB}$ ) versus $\phi$ angle in the $\theta=\pi / 2$ plane, after SC of the inputs ports, for the two prescribed MEAs in an ideal LOS scenario.

defined in Section II-A, with a ULA, the matrix $\mathrm{C}_{R_{\mathrm{LOS}}}^{1 / 2}$ can be written as

$$
\mathrm{C}_{R_{\mathrm{LOS}}}^{1 / 2}=\mathbf{W a}\left(\phi_{R}\right)
$$

and the selection diversity capacity (7) is given by

$$
C_{s c}=\log _{2}\left(1+\rho \max _{k}\left|\left(\mathbf{W a}\left(\phi_{R}\right)\right)_{k}\right|^{2}\right) .
$$

In the case that the direction of arrival of the incoming signal $\phi_{R}$ coincides with one of the directions of maximum radiation of the multidirectional-beam MEA, that is, if $\mathbf{W}_{\mathbf{i}}=1 / \sqrt{n_{T}} \mathbf{a}\left(\phi_{R}\right)^{H}$, with $\mathbf{W}_{\mathbf{i}}$ being the $i$ th row of $\mathbf{W}$, then the product $\left|\mathbf{W}_{\mathbf{i}} \mathbf{a}\left(\phi_{R}\right)\right|^{2}=\left|\mathbf{a}\left(\phi_{R}\right)^{H} \mathbf{a}\left(\phi_{R}\right)\right|^{2}=n_{R}$, which means that the signals from the receiving antennas will be coherently combined at port $i$ th of the Butler matrix. In this case, the expression for the selection diversity capacity reaches its maximum, and its value is equal to the optimal capacity of an SIMO system, given by $\log _{2}\left(1+\rho n_{R}\right)$.

Notice that the conclusions commented on here for the selection diversity capacity are also valid for the selection diversity gain.

Because the Butler matrix provides four discrete beams, and not a continuous beamforming capability, the optimum capacity will be achieved for those directions of arrival $\phi_{R}$ that coincide with the directions of maximum radiation of the four radiation patterns.

2) Performance Prediction of the Two Prescribed MEA Configurations: For the ideal LOS scenario, a more intuitive performance prediction of the two prescribed MEA can be made by inspection of the measured radiation patterns.

Fig. 9 shows the resultant directivity pattern versus the angle $\phi$ in the $\theta=\pi / 2$ plane, after SC of the input ports for each of the two prescribed MEAs, in an ideal LOS scenario. That is, for each direction of arrival, $\phi_{R}$, the radiation pattern associated to the port that provides maximum directivity on that specific direction is chosen.

As shown in Fig. 4, the multidirective-beam MEA, as a result of combining a Butler matrix with a ULA, can generate four discrete directive beams pointing at different directions in space. In an ideal LOS scenario, when the direction of arrival of the incoming signals coincides with any of the directions of maximum directivity $\left(\sim \pm 15^{\circ}, \pm \sim 50^{\circ}, \pm \sim 130^{\circ}\right.$, and $\sim \pm 165^{\circ}$ ), the multidirective-beam MEA can coherently combined the arriving signals of each antennas and take full advantage of the beamforming array gain. From Fig. 9, we observe that the resultant directivity after SC of the multidirectional-beam MEA is up to $3 \mathrm{~dB}$ larger, and never smaller, than that of the multiomnidirectional-beam MEA. Therefore, compared to a simple ULA, the multidirective-beam MEA, through beamforming, can clearly improve the SNR up to $3 \mathrm{~dB}$ and therefore improve the selection diversity gain and selection diversity capacity.

For the seek of clarifying, notice that monopole antennas over a ground plane have its direction of maximum radiation slightly above the azimuth plane. Using $n_{R}=4$ dipole antennas, which do not require a ground plane and have direction of maximum radiation exactly on the azimuth plane, one could expect improvement of up to $6 \mathrm{~dB}$ on the SNR, in theory.

As will be shown through simulations in Section V and measurements in Section VI, the proposed multidirectional-beam MEA still outperforms a simple ULA, in intermediate LOS/ NLOS scenarios, but the two prescribed MEAs tend to perform more equally as the richness of the scattering of the channel increases.

\section{Simulations}

Simulations on the selection diversity gain, selection diversity capacity, and optimum capacity have been carried out over a variety of propagation scenarios offering different angular spreads $(A S)$, K-factors $(K)$, and for different orientations of the MEAs to characterize the performance of the two prescribed MEA in intermediate channels ranging from LOS to NLOS.

Using the coordinate system shown in Fig. 1 as a reference to describe the orientation of the two prescribed MEAs, we define $\alpha$ as the angle that forms the direction of the $x$ axis with the LOS direction between the transmitter and the receiver. A representation of the angle $\alpha$ is shown in Fig. 13. Notice that the antennas in the ULA are aligned along the $y$ axis, and when $\alpha=0$, the direction of the $x$ axis is parallel to the LOS direction. $A S$ was computed as in [19], based on the central moment and without including the direct LOS component.

Unless specified, through this section, the selection diversity gain, selection diversity capacity, and optimum capacity are computed at a probability of $10 \%$, which means that during $90 \%$ of the time, the respective gain and capacity values will be above those values.

\section{A. Simulator Model}

To carry out the simulations, we have used a slow-fading frequency-nonselective channel model based on [17] and on the 
IEEE P802.11 compliant TGn MIMO channel model. ${ }^{1}$ Using this model, the channel matrix $\mathbf{h}$ can be separated into a fixed (constant, LOS) matrix $\mathbf{h}_{\mathrm{LOS}}$ and a Rayleigh (variable, NLOS) matrix $\mathbf{h}_{\mathrm{NLOS}}$

$$
\mathbf{h}=\sqrt{\frac{K}{K+1}} \mathbf{h}_{\mathrm{LOS}}+\sqrt{\frac{1}{K+1}} \mathbf{h}_{\mathrm{NLOS}}
$$

where the entries of $\mathbf{h}_{\mathrm{NLOS}}$ are correlated zero-mean unit variance, complex Gaussian random variables, and $\mathrm{K}$ is the Rician $\mathrm{K}$-factor. The entries $\mathbf{h}_{\mathbf{L O S}} \mathbf{S}_{\mathbf{k i}}$ are exponentials with argument equal to $j=\sqrt{-1}$ times the phase difference between the $k$ th transmit and $i$ th receive antenna. $\mathbf{h}$ was normalized according to [11]. Let us recall the fact that $n_{T}=1, n_{R}=4$, and the channel matrix $\mathbf{h}$ has dimensions $4 \times 1$.

To generate the Rayleigh component $\mathbf{h}_{\mathrm{NLOS}}$, the two-ring MIMO model described in [19], [20], and [21] has been used. This model has been chosen because it allows us to directly observe how the choice of antenna orientation and the shape of the antenna radiation pattern affect the system performance. The received signals are assumed to be plane waves arriving within the azimuth plane $(\theta=\pi / 2)$ and the local scatterers are assumed to be uniformly distributed within two rings, one around the transmitter and the other around the receiver. Only a single-bounce scattering has been considered. The scatterers are assigned equal scattering coefficients, with independent identically distributed uniform random phases distributed over $[0,2 \pi]$. The antennas are assumed to be isotropic, and therefore omnidirectional on the azimuth plane. Finally, the coupling between antennas has been neglected because for antenna spaced $d=0.5 \lambda$, it can be considered negligible.

Three different scenarios have been simulated.

1) Near-ideal LOS scenarios: with $K=50 \mathrm{~dB}$ and $A S \approx 0^{\circ}$. The received signal at each antenna is Rician distributed. Typical of channels with very poor scattering and a clear dominant specular path.

2) Intermediate LOS/NLOS scenario: with $K=6 \mathrm{~dB}$ and $A S \approx 30^{\circ}$. Scenario typically found in LOS indoor environments. The received signals are Rician distributed as well, with moderate scattering in the channel. To better characterize the small $A S$ of those scenarios, the ring of scatterers around the receiver was removed [19]. The radius of the ring of scatterers at the transmitter was fixed to $2500 \lambda$.

3) Rich scattered NLOS scenario: with $K=-\infty \mathrm{dB}$ and $A S \approx 90^{\circ}$. Scenario typically found in NLOS indoor environments with very rich scattering. In this case, the two rings of scatterers, around the transmitter and receiver, were used, with radius $400 \lambda$ and $2500 \lambda$, respectively.

The transmitter and the receiver were always separated by a distance equivalent to $4000 \lambda$, and the number of scatters on each ring was set to 20. The number of samples for the Monte Carlo simulation was fixed to $N=2000$ samples.

\section{B. Results on the Selection Diversity Gain}

The simulated results of this section are all based on Fig. 10, which shows the simulated selection diversity gain versus $\alpha$ for the two prescribed MEAs (multiomnidirectional-beam and

\footnotetext{
${ }^{1}$ http://www.info.fundp.ac.be/ lsc/Research/IEEE 80211-HTSG-CMSC/.
}

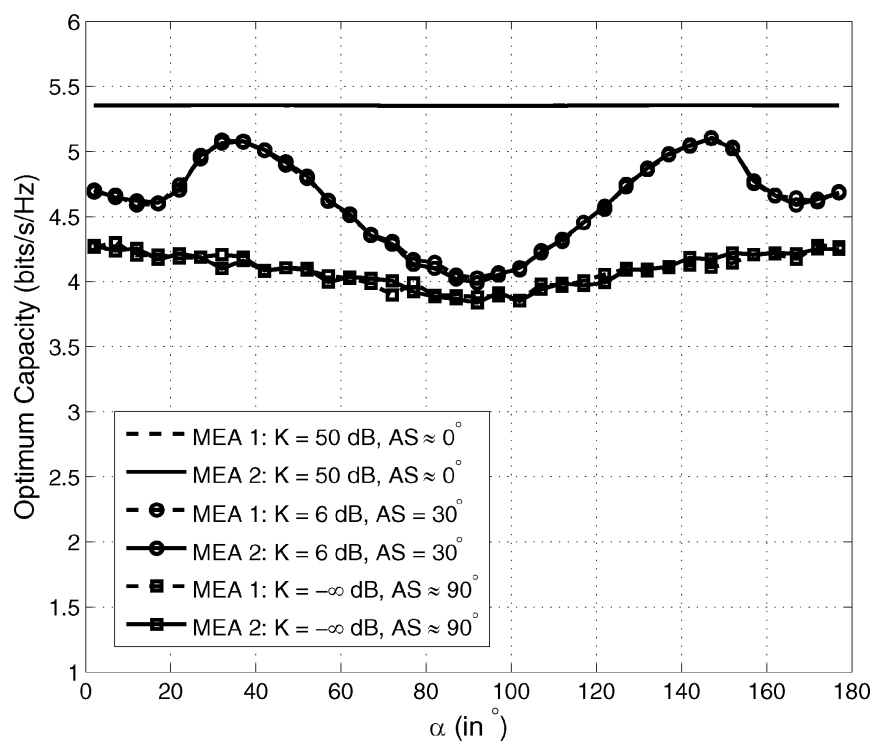

Fig. 10. Simulated selection diversity gain (in $\mathrm{dB}$ ) versus $\alpha$ for different angular spreads $\left(2^{\circ}, 20^{\circ}\right.$, and $\left.360^{\circ}\right)$ and $\mathrm{K}$-factors. MEA 1 refers to the multiomnidirectional-beam MEA and MEA 2 refers to the multidirectional-beam MEA using an ideal Butler matrix.

multidirectional-beam) and for the three scenarios described in Section V-A.

From Fig. 10, one can observe that in the near-ideal LOS scenario, where the specular component is the dominant (large- $\mathrm{K}$ factor) and the $A S$ is very small, $A S \approx 0^{\circ}$, an improvement of approximately $2.5-6 \mathrm{~dB}$ on the SNR is achieved with the proposed multidirectional-beam MEA with respect to a simple ULA, for different directions of arrival $\alpha$ of the incoming signals. This can be easily explained from the fact that with poor scattering, the arriving rays are highly correlated in phase and magnitude, and coherent combining techniques such as beamforming can take advantage of it. In fact, the beamforming gain generated by the combined use of the ULA and the Butler matrix is responsible for the improvement on the SNR. On the other hand, the large correlation among the signals at each receiving antenna degrades the achievable selection diversity gain of a simple ULA, as seen in Fig. 10, that is always around $0 \mathrm{~dB}$. Notice that the simulations agree well the theory commented on in Section IV-B.

Notice that in Fig. 10 one can clearly distinguish the shape of the four directive radiation patterns for the multidirective-beam MEA in those scenarios with small $A S$.

In the intermediate LOS/NLOS scenarios with $A S=30^{\circ}$ and $K=6 \mathrm{~dB}$, although the multidirectional-beam MEA still outperforms a simple ULA, their curves tend to unite. This tendency becomes more obvious as the $A S$ further increases and the $\mathrm{K}$-factor reduces $\left(A S \approx 90^{\circ}\right.$ and $\left.K=-\infty \mathrm{dB}\right)$. This can be explained as follows. As the richness of the scattering increases, so does the $A S$, and the arriving rays are less correlated. In those situations, coherent techniques such as beamforming lose their advantage and the benefits of signal diversity increase. On the other hand, due to the fact that the multidirective-beam MEA simultaneously takes advantage of both techniques, it always outperforms the multiomnidirectional-beam MEA, which only takes advantage of the signal diversity. 
In the rich scattered NLOS scenario with an $A S=90^{\circ}$ and $K=-\infty \mathrm{dB}$, the curves for the multiomnidirectional-beam and multidirectional-beam MEAs are superimposed. As commented on in Section IV-A, this can be explained from the fact that in NLOS scenarios, a very wide range of directions of arrival are approximately equiprobable, which makes the shape of the radiation pattern irrelevant, as long as the total captured radiation energy is the same and the pattern orthogonality is preserved. Notice that the simulated selection diversity gain is approximately $9 \mathrm{~dB}$, which agrees well with the theoretical selection diversity gain at a probability of $10 \%$ by combining four branches in independent Rayleigh channels using SC [13]. In the limit case of an ideal NLOS scenario with $A S \approx 360^{\circ}$, one would expect the two curves to be perfectly superimposed and around $9 \mathrm{~dB}$ for any value of $\alpha$.

From the above figures, one can observe that the signal diversity is a major contributor to the increase of the SNR with respect to beamforming, as the $A S$ becomes similar or larger to the beamwidth $B W \approx 30^{\circ}$ of the radiation patterns formed by the combined use of the Butler matrix and the ULA.

As a conclusion, the proposed multidirective-beam MEA does not degrade the selection diversity gain in rich scattering environments with respect to a simple ULA, while it dramatically improves the SNR in Rician channels (LOS scenarios) with small $A S$. Notice that the above conclusion is in agreement with the predictions in Section IV.

Finally, notice that a minimum in the diversity gain is observed at $\alpha=90^{\circ}$ for the two prescribed MEA. This corresponds to the case where the receiving antennas of the ULA are aligned with the LOS direction, where the correlation of the arriving rays reaches a maximum. In addition, this direction coincides with that of smallest beamforming gain.

\section{Results on the Selection Diversity Capacity and Optimal Capacity}

Figs. 11 and 12 show the simulated selection diversity capacity and optimum capacity, respectively, versus $\alpha$ for the two prescribed MEA (multiomnidirectional-beam and multidirectional-beam MEAs) and for the three scenarios described in Section V-A. Assume an SNR $=10 \mathrm{~dB}$.

Because the optimum capacity given by (6) does not impose any constraint in the linear transformation of the antenna outputs at the receiver [1], one would expect that independently of the $A S$ and direction of arrival $\alpha$, the two prescribed MEA configurations achieve exactly the same capacity. That is, one should see that the hardware processing of the Butler matrix does not impact the optimum capacity. This fact is corroborated in Fig. 12 where the curves for the two MEAs are always superimposed. This information will be used here as a reference for how far the achieved selection diversity capacity is from the optimum capacity using MRC.

From Fig. 12, we also observe that the optimum capacity, at a probability of $10 \%$, decreases as the richness of the scattering increases. This can be explained from the fact that in a rich multipath environment, the signal fading is large, and for a fixed number of transmitting $\left(n_{T}=1\right)$ and receiving antennas $\left(n_{R}=4\right)$, this fading is responsible for an increase of the probability of smaller capacity values. This is especially severe at a

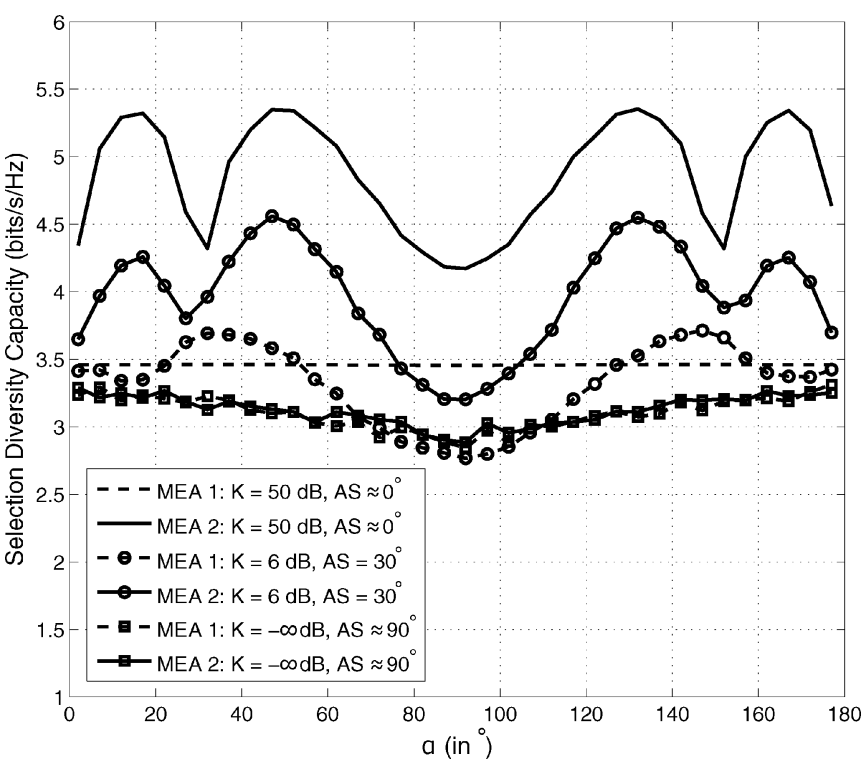

Fig. 11. Simulated selection diversity capacity (in bits/s/Hz) versus $\alpha$ for different angular spreads $\left(2^{\circ}, 20^{\circ}\right.$, and $\left.360^{\circ}\right)$ and $\mathrm{K}$-factors. MEA 1 refers to the multiomnidirectional-beam MEA and MEA 2 refers to the multidirectionalbeam MEA using an ideal Butler matrix.

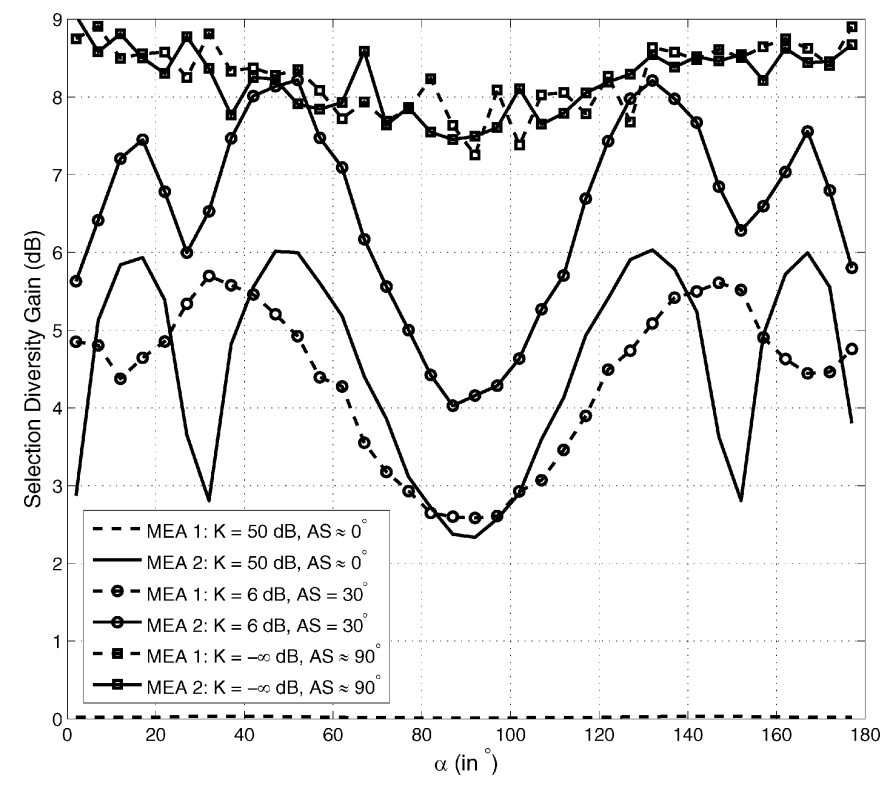

Fig. 12. Simulated optimum capacity (in bits/s/Hz) versus $\alpha$ for different angular spreads $\left(2^{\circ}, 20^{\circ}\right.$, and $\left.360^{\circ}\right)$ and $\mathrm{K}$-factors. MEA 1 refers to the multiomnidirectional-beam MEA and MEA 2 refers to the multidirectional-beam MEA using an ideal Butler matrix.

probability of $10 \%$. This effect will be shown later through measurements in Figs. 17 and 18 in Section VI.

For the rich scattered NLOS scenario, the selection diversity gain is upper bounded by the optimum capacity value of approximately $4.3 \mathrm{bits} / \mathrm{s} / \mathrm{Hz}$. On the other hand, in the near-ideal LOS scenario, it is upper bounded by $5.35 \mathrm{bits} / \mathrm{s} / \mathrm{Hz}$. Notice that the $5.35 \mathrm{bits} / \mathrm{s} / \mathrm{Hz}$ agrees well with the capacity of an equivalent SISO channel with a SNR of $16 \mathrm{~dB}$, as a result of adding $6 \mathrm{~dB}$ from the Butler matrix beamforming gain (array factor) to the original SNR of $10 \mathrm{~dB}$. 
From Fig. 11, we observe that the selection diversity capacity follows the exact same trends as the selection diversity gain shown in Fig. 10 and commented on in detail in Section V-B. This can be explained from the fact that the selection diversity gain and the selection diversity capacity are two different figures of merit used to evaluate essentially the same idea, the SC performance. Interestingly, and in particular for the rich scattered NLOS scenario, the achieved selection diversity capacity is around $3.3 \mathrm{bits} / \mathrm{s} / \mathrm{Hz}$, about $1 \mathrm{bit} / \mathrm{s} / \mathrm{Hz}$ away from the optimal capacity using MRC. On the other hand, in those scenarios with small $A S\left(A S \approx 0^{\circ}, 30^{\circ}\right)$, we also observe that the multidirectional-beam MEA is clearly improving the selection diversity capacity with respect to a simple ULA. In fact, for the near-ideal LOS scenario at certain values of $\alpha\left(\sim \pm 15^{\circ}\right.$, $\pm \sim 50^{\circ}, \pm \sim 130^{\circ}$, and $\sim \pm 165^{\circ}$ ) that coincide with the directions of maximum directivity of the multidirectional-beam MEA, the selection diversity capacity is equal to the optimum capacity of $5.35 \mathrm{bits} / \mathrm{s} / \mathrm{Hz}$.

For the same reason as those commented on in Section V-B, the optimum capacity reaches a minimum at $\alpha=90^{\circ}$.

The above conclusions are also in agreement with the predictions in Section IV.

\section{MEASUREMENT}

Measurements on selection diversity gain, selection diversity capacity, and optimum capacity were conducted to validate the results shown in previous simulations and analysis.

Unless specified, the values of selection diversity gain, selection diversity capacity, and optimum capacity are referred to those computed at a probability of $10 \%$.

\section{A. Description of the Measurement Setup}

The measurements were conducted using two robotic arms separated $4 \mathrm{~m}$ approximately. A transmitting monopole moving at steps of $0.8 \lambda$ was installed in one of the two robotic arms, while the two prescribed MEA configurations under test were installed on the other arm, and moved at steps of $0.1 \lambda$. On each measurement, the number of recollected samples was $N=$ 1000. This setup allowed us to perform repetitive measurements and compare the two prescribed MEAs among different MIMO propagation scenarios. A picture of one of the robotic arms is shown in Fig. 14.

The measurements were conducted by transmitting a single tone at $2.45 \mathrm{GHz}$ and using a network analyzer connected to the transmitting and receiving antennas, to obtain the values of the entries of the channel matrix $\mathbf{h}$.

Measurements were carried out in two different scenarios.

1) Near-ideal LOS scenario: was reproduced inside an anechoic chamber. Due to the absorbing material on the walls of the anechoic chamber, only a direct LOS propagation path existed between the transmitter and the receiver.

2) Rich scattered NLOS scenario: reproduced in a room of approximately $9 \times 4 \mathrm{~m}$ with several metallic shields, tables, and other objects on it. The layout of the room is shown in Fig. 13. There was no direct visibility between the transmitter and the receiver.

Those two scenarios were chosen as limit cases of real propagation environments.

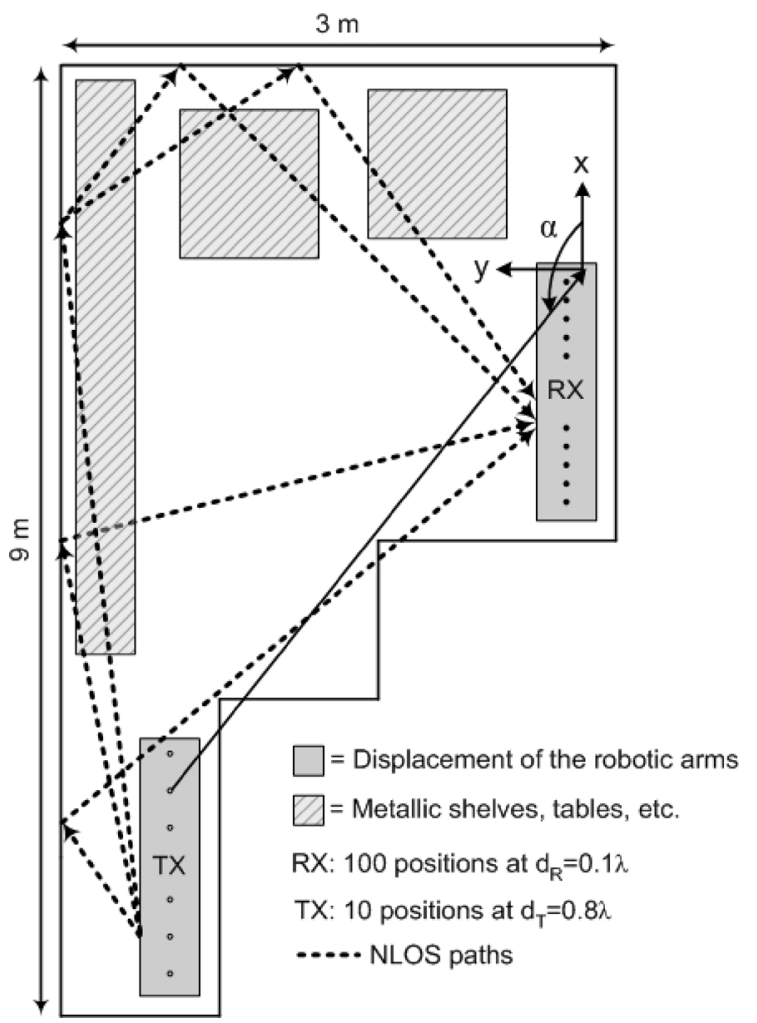

Fig. 13. Layout and dimensions of the room where the NLOS measurements were conducted. The LOS measurements were conducted inside an anechoic chamber.

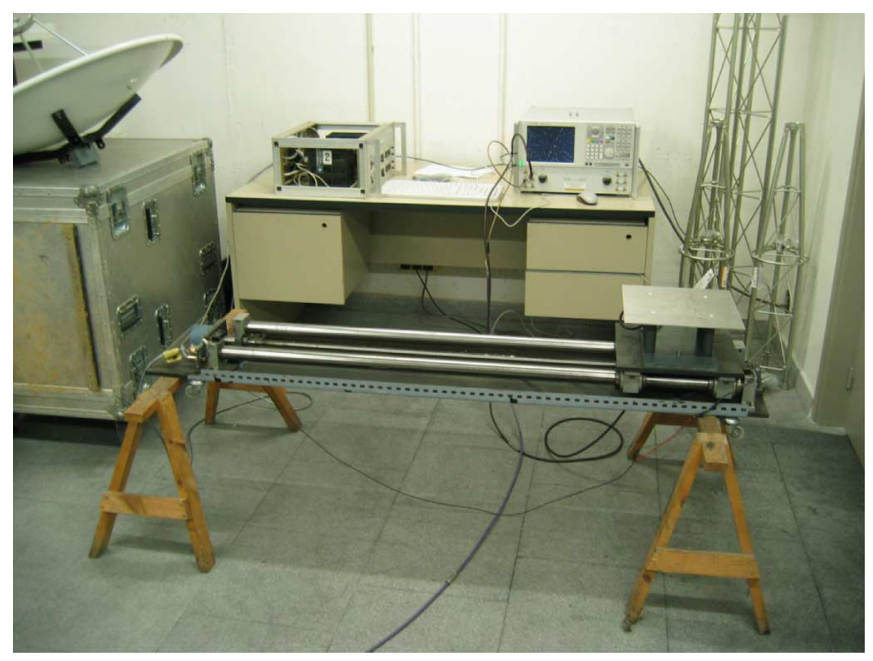

Fig. 14. Picture of one of the two automatized robotic arms used in the measurement setup, with the ULA mounted on it.

\section{B. Results on Selection Diversity Gain}

Figs. 15 and 16 show, for the LOS and NLOS scenarios, respectively, the measured cdfs of the normalized received power on each accessible port and after selection combining, for the two prescribed MEA configurations. In addition, the cdf curves for an ideal MEA combining a ULA and an ideal lossless Butler matrix are also included. For this last case, the curves were obtained by postweighting the measured signals at the output of the ULA by the transformation vectors $\mathbf{W}$ of an ideal Butler matrix. 


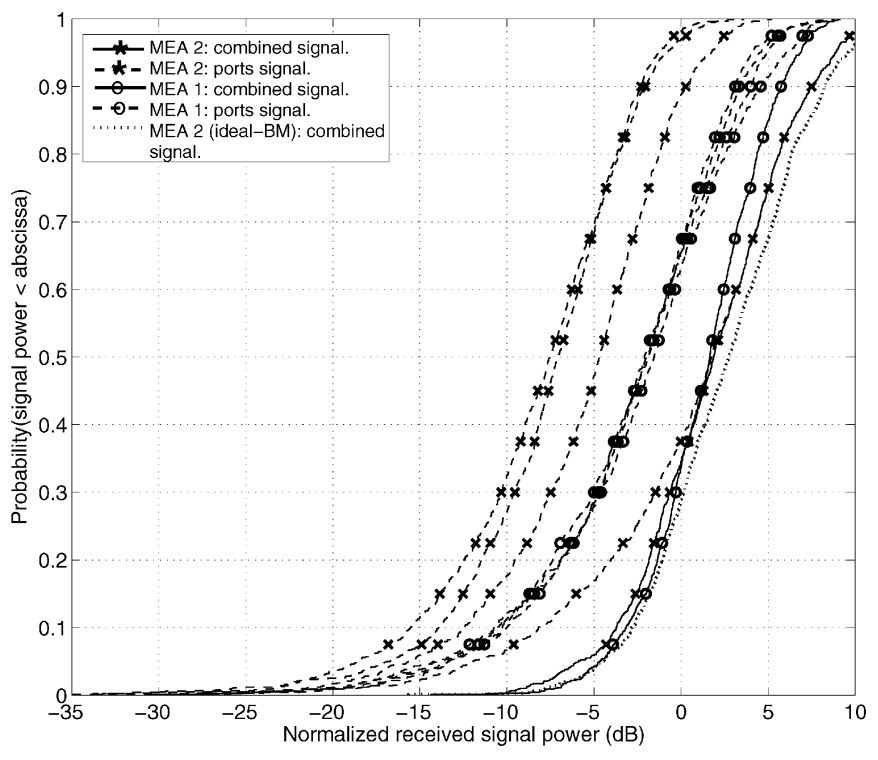

Fig. 15. CDF curves of the normalized received power on each accessible port (port signal) and after selection combining (combined signal), in the NLOS scenario, for the two prescribed MEAs. MEA 1 refers to the multiomnidirectional-beam MEA and MEA 2 refers to the multidirectional-beam MEA using a real Butler matrix.

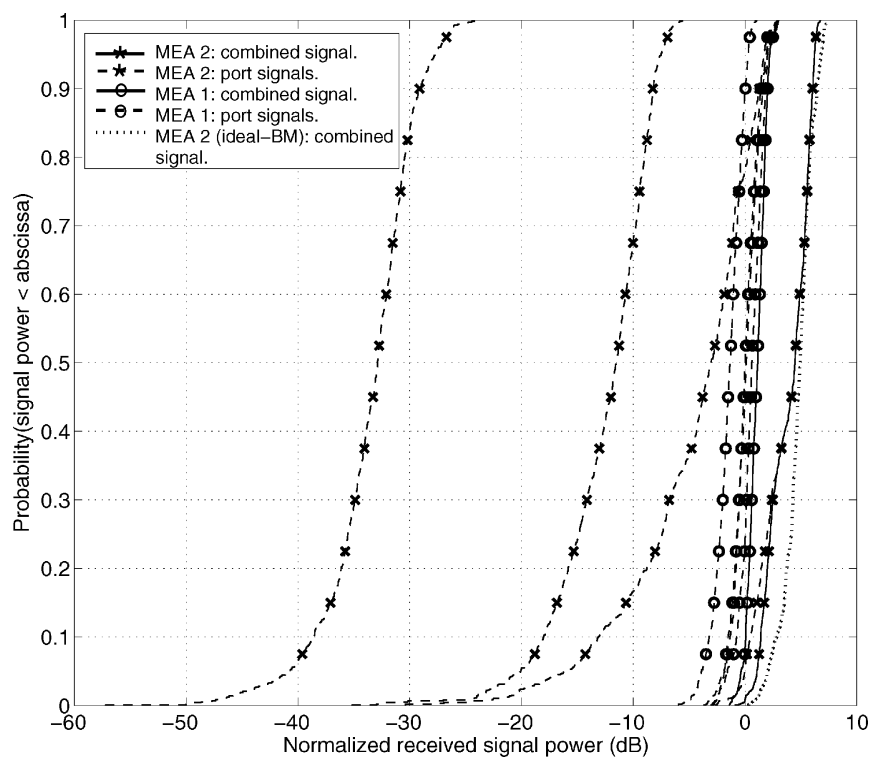

Fig. 16. CDF curves of the normalized received power on each accessible port (port signal) and after selection combining (combined signal), in the LOS scenario, for the two prescribed MEAs. MEA 1 refers to the multiomnidirectionalbeam MEA and MEA 2 refers to the multidirectional-beam MEA using a real Butler matrix.

For the NLOS measurements shown in Fig. 15, we observe that the selection diversity gain is approximately equal for the two prescribed MEA configurations: $6.29 \mathrm{~dB}$ for the multidirective-beam and $6.84 \mathrm{~dB}$ for the multiomnidirectional-beam MEA, respectively. In fact, the cdf curves after SC for the two prescribed MEA are approximately superimposed. This is in agreement with the results obtained from simulations in a rich scattered NLOS scenario with $A S \approx 90^{\circ}$ and $k=-\infty \mathrm{dB}$, shown in Section V-B, and from the correlation coefficients analysis presented in Section IV-A. Once again we observe that, in rich scattering environments, the shape of the patterns does not impact the diversity gain, as long as the total captured radiation energy is the same and the patterns are orthogonal to each other. Remember that from Section IV-A this is equivalent to having decorrelated input ports for the MEA.

Notice, however, that the selection diversity gain achieved with the multidirectional-beam MEA is $0.55 \mathrm{~dB}$ smaller than that achieved with the simple ULA. This can be explained from the fact that the Butler matrix has approximately $0.8 \mathrm{~dB}$ of insertion loss. This also explains, in Fig. 15, the small shift to the left of the cdf curve for the MEA using the real Butler matrix, with respect to the cdf curve of the MEA using an ideal Butler matrix. By looking at the average SNR after SC, one can observe that the impact of the Butler matrix losses at those probabilities is smaller. In fact, looking at the mean, multidirectional-beam MEA outperforms by $0.22 \mathrm{~dB}$ a simple ULA, even considering the losses of the external circuitry.

On the other hand, using the multiomnidirectional-beam MEA, the four antennas receive similar power levels, while in the case of the multidirective-beam MEA, one of the ports is favored in detriment of the other three. This provides an additional SNR margin (computed at a probability of $10 \%$ ) of $1.75 \mathrm{~dB}$ in one of the ports, with respect to the multiomnidirectional-beam MEA. This can be explained from the fact that in non-perfect NLOS scenarios, there normally exist several dominant directions of arrival, and the beamforming array gain can take advantage of it. This is particularly advantageous for the detection of signal in low SNR conditions.

For the near-ideal LOS measurements shown in Fig. 16, we observe that the diversity gain after SC for the multidirectivebeam MEA is $2.14 \mathrm{~dB}$, while it is $0.78 \mathrm{~dB}$ for the multiomnidirectional-beam MEA. The proposed multidirectional-beam MEA improves the SNR by about $1.36 \mathrm{~dB}$ thanks to the beamforming gain obtained from the combined use of a Butler matrix and a ULA. This improvement becomes more obvious when looking at the mean SNR, which is improved by approximately $3.3 \mathrm{~dB}$. These results are also in agreement with the simulations shown in Section V-B and correlation analysis in Section IV-A.

Similarly as in the NLOS case, the received power level by one of the ports of the multidirective-beam MEA is significantly larger than in the other three ports. However, as seen in Fig. 16, in the near-ideal LOS case, the differences in the received power level among the different branches are much larger. This can be explained from the fact that for this particular measurement arrangement, the directions of arrival of the incoming rays fall within the beamwidth of one of the four directive beams, while at the same time the directions correspond to those of secondary lobes or nulls in the radiation patterns of the other three beams. Notice that, in scenarios with small $A S$, the coherent beamforming is the major contributor, compared to signal diversity, to the increase in the SNR.

Table I summarizes the results on selection diversity gain for the LOS and NLOS cases. Notice that the realized gains include the losses of the Butler matrix. Comparative values with the case using an ideal lossless Butler matrix are included to show the potential gains that one would obtain with a low-loss design of the Butler matrix. 
TABLE I

SELECTION Diversity Gains For THE Two MEA CONFIGURATIONS IN THE LOS AND NLOS CASE

\begin{tabular}{|c||c|c|c||c|c|c|}
\hline \multicolumn{1}{|c||}{} & \multicolumn{3}{c||}{ NLOS } & \multicolumn{3}{c|}{ LOS } \\
\hline & \multicolumn{3}{c|}{ Selection Diversity } & \multicolumn{3}{c|}{ Selection Diversity } \\
\hline & $\begin{array}{c}\text { MEA 1: } \\
\text { no-BM }\end{array}$ & $\begin{array}{c}\text { MEA 2: } \\
\text { real-BM }\end{array}$ & $\begin{array}{c}\text { ideal } \\
\text { BM }\end{array}$ & $\begin{array}{c}\text { MEA 1: } \\
\text { no-BM }\end{array}$ & $\begin{array}{c}\text { MEA 2: } \\
\text { real-BM }\end{array}$ & $\begin{array}{c}\text { ideal } \\
\text { BM }\end{array}$ \\
\hline $10 \%$ & 6.84 & 6.29 & 7.18 & 0.78 & 2.14 & 3.57 \\
\hline mean & 3.22 & 3.44 & 4.30 & 1.73 & 5.06 & 5.56 \\
\hline
\end{tabular}

\section{Results on the Selection Diversity Capacity and Optimal Capacity}

Figs. 17 and 18 show, for the NLOS and LOS scenarios, respectively, the measured cdf of the selection diversity capacity and optimum capacity for the two prescribed MEA configurations. In addition, the cdf curves for an ideal MEA combining a ULA and an ideal lossless Butler matrix are also included. Assume an $\mathrm{SNR}=10 \mathrm{~dB}$.

Because selection diversity gain and selection diversity capacity are two different figures of merit used to evaluate the performance of the same combining technique, the results shown in Figs. 17 and 18 about the selection diversity capacity follow the same exact trends as those in Figs. 15 and 16 about the selection diversity gain.

For the rich scattered NLOS measurements shown in Fig. 17, one can observe that the two prescribed MEAs perform similarly, as predicted also in Sections IV and V. In fact, the selection diversity capacity is 2.54 and $2.39 \mathrm{bits} / \mathrm{s} / \mathrm{Hz}$ for the multiomnidirectional-beam and multidirectional-beam MEA, respectively. Once again, the $0.8 \mathrm{~dB}$ of insertion losses of the Butler matrix impacts negatively on the selection diversity capacity. In fact, notice that in the case of having and ideal Butler matrix, the predicted selection diversity capacity would be approximately $2.63 \mathrm{bits} / \mathrm{s} / \mathrm{Hz}$. Finally, in the NLOS scenario, the selection diversity capacity is only $0.88 \mathrm{bits} / \mathrm{s} / \mathrm{Hz}$ below the optimum capacity value, which agrees well with the simulations results on Section V-C. The mean values of the selection diversity capacity follow the same trend as those computed at a probability of $10 \%$.

For the near-ideal LOS measurements shown in Fig. 18, the cdf curves of the selection diversity capacity for the two prescribed MEAs do not coincide, and in fact the two MEAs configurations perform very different. The selection diversity capacity cdf curve for the multidirectional-beam MEA is well to the right with respect to that of the multiomnidirectional-beam MEA, meaning that a larger selection diversity capacity is achieved with the multidirectional-beam MEA at all probabilities. The improvement in the selection diversity capacity is approximately $0.42 \mathrm{bits} / \mathrm{s} / \mathrm{Hz}$, which agrees well with the increment of $1.36 \mathrm{~dB}$ in the SNR commented on in Section VI-B, due to the beamforming gain achieved from the combined use of the Butler matrix and a ULA. Looking at the average values, the differences become more obvious, and the multidirective-beam MEA outperforms the multiomnidirectional-beam MEA by $0.91 \mathrm{bits} / \mathrm{s} / \mathrm{Hz}$. Notice also that using an ideally lossless Butler matrix, the performance improvement would be even larger and approximately of $1 \mathrm{bit} / \mathrm{s} / \mathrm{Hz}$. This raises the interest in the design of low-loss Butler matrices.

Tables III and II summarize the results of the optimum and selection diversity capacity [1] for the LOS and NLOS case, respectively. Notice that in general, at a probability of $10 \%$, the

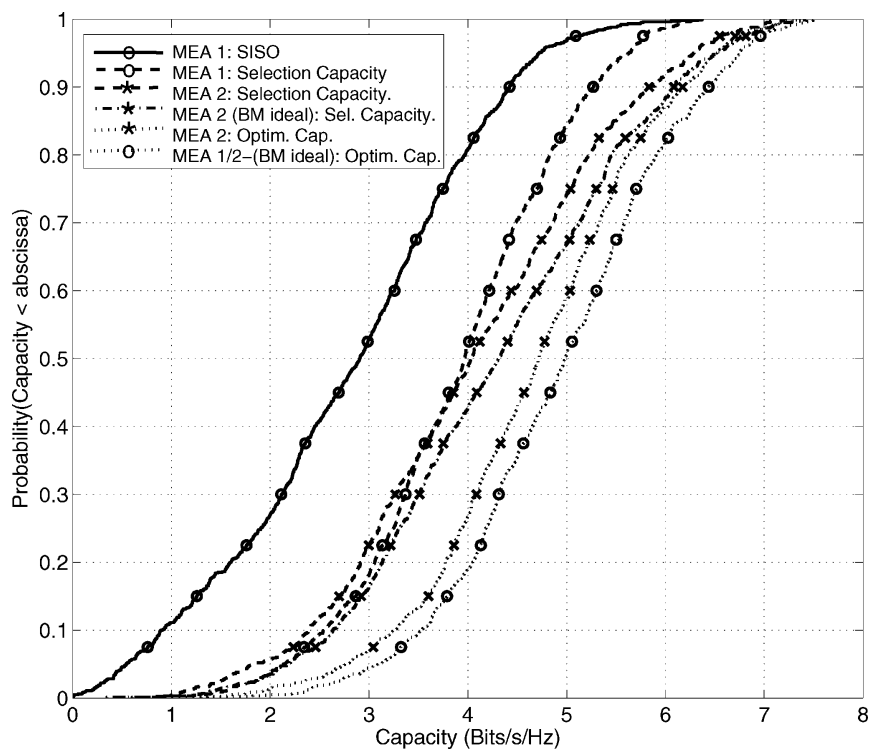

Fig. 17. CDF curves of the selection diversity capacity and optimum capacity, in the NLOS scenario, for the two prescribed MEAs, at an SNR $=10 \mathrm{~dB}$. MEA 1 refers to the multiomnidirectional-beam MEA and MEA 2 refers to the multidirectional-beam MEA using a real Butler matrix.

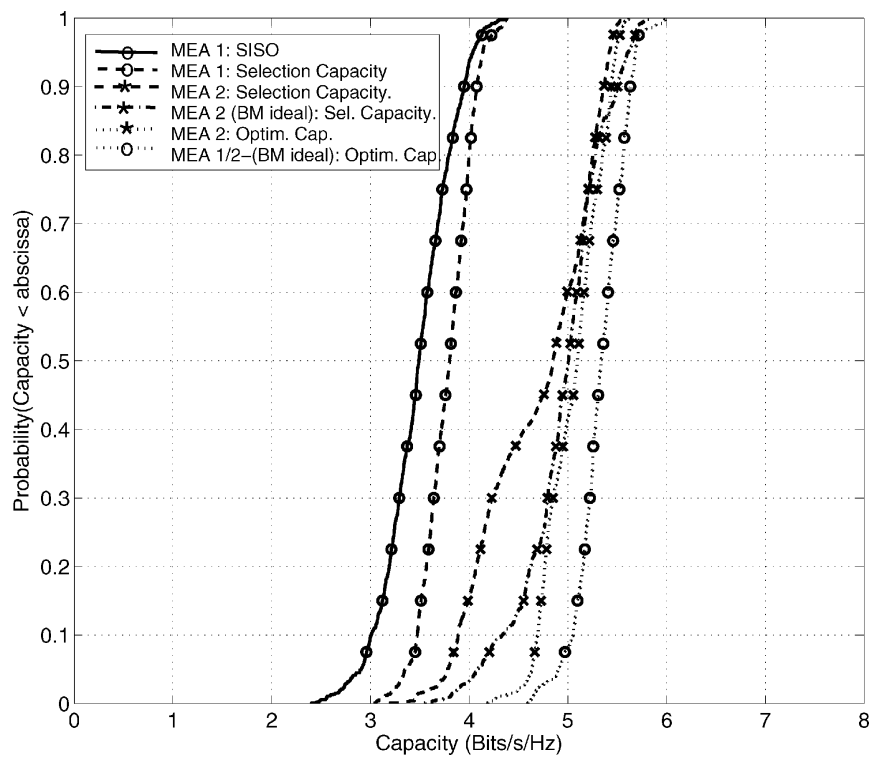

Fig. 18. CDF curves of the selection diversity capacity and optimum capacity, in the LOS scenario, for the two prescribed MEAs, at a $S N R=10 \mathrm{~dB}$. MEA 1 refers to the multiomnidirectional-beam MEA, and MEA 2 refers to the multidirectional-beam MEA using a real Butler Matrix.

capacity values of the LOS case are larger than those for the NLOS case. As commented also in Section V-C, this can be explained from the fact that in an NLOS scenario, the existence of fading increases the probability of the smaller capacity values.

Because the optimum capacity given by (6) does not impose any constraint in the linear transformation of the antenna outputs at the receiver, the optimum capacity cdf curves for the two prescribed MEAs should be superimposed. However, a small shift can be observed among the two curves, in Figs. 17 and 18, which can be explained from the $0.8 \mathrm{~dB}$ of insertion loss of the Butler matrix.

Once again, through measurements, it has been demonstrated that the proposed MEA consisting of a ULA combined with a 
TABLE II

SELECTION DIVERSITY CAPACITY AND OPTIMAL CAPACITY (BITS/S/Hz) FOR THE Two MEA CONFIGURATIONS IN THE NLOS CASE

\begin{tabular}{|c||c|c|c|c|c|c|}
\hline \multicolumn{1}{|c||}{} & \multicolumn{6}{c|}{ NLOS } \\
\hline \multicolumn{1}{|c||}{} & \multicolumn{3}{c|}{ SEL. Cap. } & \multicolumn{3}{c|}{ OPT. Cap. } \\
\hline & MEA 1: & MEA 2: & Ideal & MEA 1: & MEA 2: & Ideal \\
no-BM & BM & BM & no-BM & BM & BM \\
\hline $10 \%$ & 2.54 & 2.39 & 2.63 & 3.54 & 3.27 & 3.54 \\
\hline mean & 3.92 & 4.07 & 4.31 & 4.95 & 4.69 & 4.95 \\
\hline
\end{tabular}

TABLE III

SELECTION DIVERSITY CAPACITY AND OPTIMAL CAPACITY (BITS/s/Hz) FOR THE Two MEA CONFIGURATIONS IN THE LOS CASE

\begin{tabular}{|c||c|c|c|c|c|c|}
\hline \multicolumn{1}{|c||}{} & \multicolumn{6}{c|}{ LOS } \\
\hline \multicolumn{1}{|c||}{} & \multicolumn{3}{c|}{ SEL. Cap. } & \multicolumn{3}{c|}{ OPT. Cap. } \\
\hline & $\begin{array}{c}\text { MEA 1: } \\
\text { no-BM }\end{array}$ & $\begin{array}{c}\text { MEA 2: } \\
\text { BM }\end{array}$ & $\begin{array}{c}\text { Ideal } \\
\text { BM }\end{array}$ & $\begin{array}{c}\text { MEA 1: } \\
\text { no-BM }\end{array}$ & $\begin{array}{c}\text { MEA 2: } \\
\text { BM }\end{array}$ & $\begin{array}{c}\text { Ideal } \\
\text { BM }\end{array}$ \\
\hline $10 \%$ & 3.47 & 3.89 & 4.34 & 5.05 & 4.69 & 5.05 \\
\hline mean & 3.78 & 4.69 & 4.95 & 5.33 & 5.06 & 5.33 \\
\hline
\end{tabular}

Butler matrix improves the selection diversity capacity performance with respect to a simple ULA in LOS conditions, and performs equally well than a ULA in NLOS scenarios. The measurements agree will with the simulations in Section $\mathrm{V}$ and performance analysis in Section IV.

Notice also that for both LOS and NLOS scenarios, the selection diversity capacity, despite being a suboptimal technique and its simplicity of implementation, provides a significant improvement on the capacity of the system with respect to the SISO system.

\section{CONCLUSION}

An optimized linear multielement antenna for selection combining schemes that improves the selection diversity gain and selection diversity capacity in medium and low multipath environments with respect to a simple uniform linear array has been proposed. The proposed MEA performs equally as well as a ULA in highly scattered environments. This improvement is realized even considering the losses of the Butler matrix network. The performance has been evaluated in terms of selection diversity gain and selection diversity capacity through the analysis of the correlation coefficients, simulations, and measurements. The use of multidirective-beam antennas can also increase the SNR margin at the receiver ports, which is a beneficial feature for the detection of the signal in low SNR regime.

\section{REFERENCES}

[1] G. Foschini and M. J. Gans, "On limits of wireless communications in a fading environment when using multiples antennas," Wireless Personal Commun., vol. 6, pp. 331-335, 1998.

[2] I. E. Telatar, "Capacity of multiantenna Gaussian channels," Eur. Trans. Telecommun., vol. 10, no. 6, pp. 585-595, Oct. 1999, Bell Labs, Lucent Technologies, 1995 Tech. Memo..

[3] D. G. Brennan, "Linear diversity combing techniques," Proc. IRE, vol. 47, pp. 1075-1101, Jun. 1959.

[4] M. Kavehrad and P. J. Mclane, "Performance of low-complexity channel coding and diversity for spread spectrum in indoor, wireless communications," ATT Tech. J., vol. 64, no. 8, Oct. 1985.

[5] Y. Chen and C. Tellambura, "Output in equally correlated Rayleigh, Rician, and Nakagami-m fading channels," IEEE Trans. Commun., vol. 52, pp. 1948-1959, Nov. 2004.

[6] A. Lozano, F. R. Farrokhi, and R. A. Valenzuela, "Asymptotically optimal open-loop space-time architecture adaptive to scattering conditions," in Vehicular Technol. Conf. 2001, May 2001, vol. 6-9, pp. 73-77.
[7] J. L. Allen, "A theoretical limitation on the formation of lossless multiple beams in linear arrays," IEEE Trans. Antennas Propag., vol. AP-9, no. 4, pp. 350-352, 1961.

[8] W. K. Kahn and H. Kurss, "The uniqueness of the lossless feed network for a multibeam array," IEEE Trans. Antennas Propag., vol. AP-10, pp. 100-101, Jan. 1962.

[9] A. F. Molisch and X. Zhang, "FFT-Based hybrid antenna selection schemes for spatially correlated MIMO channels," IEEE Commun. Lett., vol. 8, p. 38, Jan. 2004.

[10] C. Collado, A. Grau, and F. D. Flaviis, "Dual-band butler matrix for WLAN systems," in IEEE Int. Microwave Symp. (MTT-S), 2005.

[11] M. A. Jensen and J. W. Wallace, "A review of antennas and propagation for MIMO wireless communications," IEEE Trans. Antennas Propag. pp. 2810-2824, Nov. 2004.

[12] A. Cardama, L. Jofre, J. M. Rius, J. Romeu, and S. Blanch, Antenas, 1st ed. Barcelona, Spain: Edicions UPC, 1998.

[13] W. C. Jakes, Microwave Mobile Commun., 1974.

[14] D. Chizhik, F. Rashid-Farrokhi, J. Ling, and A. Lozano, "Effect of antenna separation on the capacity of BLAST in correlated channels," IEEE Commun. Lett., vol. 4, pp. 337-339, 2000.

[15] S. Blanch, J. Romeu, and I. Corbella, "Exact representation of antenna system diversity performance from input parameter description," Electron. Lett., vol. 39, no. 9, pp. 705-707, May 2003.

[16] D. M. Pozar, Microwave Engineering, 2nd ed. New York: Wiley, 1998.

[17] P. Soma, D. Baum, V. Erceg, R. Krishnamoorthy, and A. Paulraj, "Analysis and modeling of multiple-input multiple-output (MIMO) radio channel based on outdoor measurements conducted at $2.5 \mathrm{GHz}$ for fixed BWA applications," in IEEE Int. Conf. Commun. 2002 (ICC 2002) , May 2002, vol. 1, pp. 272-276.

[18] F. Farrokhi, A. Lozano, G. Foschini, and R. Valenzuela, "Spectral efficiency of FDMA/TDMA wireless systems with transmit and receive antenna arrays," IEEE Trans. Wireless Commun., vol. 1, pp. 591-599, Oct. 2002.

[19] P. Petrus, J. H. Reed, and T. S. Rappaport, "Geometrical-based statistical macrocell channel model for mobile environments," IEEE Trans. Commun., vol. 50, pp. 495-502, Mar. 2002.

[20] S. Wang, K. Raghukumar, A. Abdi, J. Wallace, and M. Jensen, "Indoor MIMO channel: A parametric correlation model and experiments results," in 2004 IEEE Sarnoff Symp. Adv. Wired Wireless Commun., Apr. 2004, pp. 1-5

[21] D.-S. Shiu, G. J. Foschini, M. J. Gans, and J. M. Kahn, "Fading correlation and its effect on the capacity of multielement antenna systems," IEEE Trans. Commun., vol. 48, pp. 502-513, Mar. 2000.

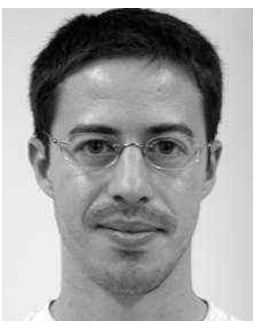

Alfred Grau was born in Barcelona, Spain, in 1977. $\mathrm{He}$ received the telecommunication engineer degree from the Universitat Politècnica de Catalunya (UPC), Barcelona, Spain, and the M.S. degree from the University of California, Irvine, in 2001 and 2004, respectively, where he is currently pursuing the Ph.D. degree.

His current research is focused on compact antennas and reconfigurable multielement antennas for MIMO communication systems.

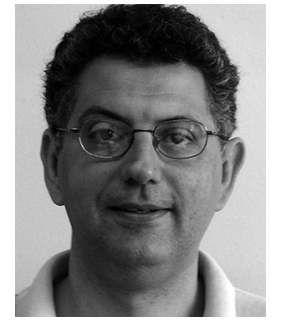

Jordi Romeu was born in Barcelona, Spain, in 1962 $\mathrm{He}$ received the ingeniero de telecomunicación and doctor ingeniero de telecomunicación degrees from the Universitat Politècnica de Catalunya (UPC), Barcelona, Spain, in 1986 and 1991, respectively.

In 1985, he joined the Photonic and Electromagnetic Engineering Group, Signal Theory and Communications Department, UPC. Currently, he is a full Professor there, where he is engaged in research in antenna near-field measurements, antenna diagnostics, and antenna design. He was a Visiting Scholar at the Antenna Laboratory, University of California, Los Angeles, in 1999, on a NATO Scientific Program Scholarship, and in 2004 at University of California, Irvine. He has received several patents and has published 35 refereed papers in international journals and 50 conference proceedings.

Dr. Romeu was Grand Winner of the European IT Prize in 1998, awarded by the European Commission, for his contributions to the development of fractal antennas. 


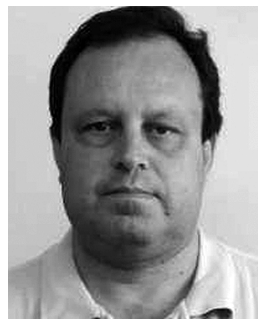

Sebastián Blanch was born in Barcelona, Spain, in 1961. He received the ingeniero and doctor ingeniero degrees in telecommunication engineering from the Polytechnic University of Catalonia (UPC), Barcelona, Spain, in 1989 and 1996, respectively.

In 1989, he joined the Electromagnetic and Photonics Engineering Group, Signal Theory and Communications Department, UPC. Currently, he is an Associate Professor at UPC. His research interests are antenna near-field measurements, antenna diagnostics, and antenna design.

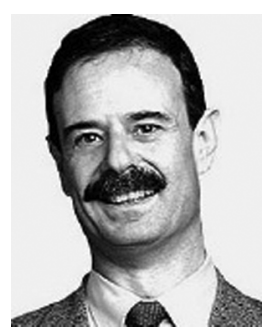

Lluis Jofre (M'78) was born in Barcelona, Spain, in 1956. He received the M.Sc. (Ing.) and Ph.D. (Dr. Ing.) degrees in electrical engineering (telecommunications engineering) from the Technical University of Catalonia (UPC), Barcelona, in 1978 and 1982, respectively.

From 1979 to 1980, he was a Research Assistant with the Electrophysics Group, UPC, where he worked on the analysis and near-field measurement of antenna and scatterers. From 1981 to 1982, he was with the Ecole Superieure d'Electricite, Paris, France, where he was involved in microwave antenna design and imaging techniques for medical and industrial applications. In 1982, he became an Associate Professor with the Communications Department, Telecommunication Engineering School, UPC, where he became a full Professor in 1989. From 1986 to 1987, he was a Visiting Fulbright Scholar at the Georgia Institute of Technology, Atlanta, working on antennas and electromagnetic imaging and visualization. From 1989 to 1994, he was Director of the Telecommunication Engineering School, and from 1994 to 2000, he was UPC Vice-Rector for Academic Planning. His research interests include antennas, scattering, electromagnetic imaging, and wireless communications. He has published more than 100 scientific and technical papers, reports, and chapters in specialized volumes. During 2000 and 2001, he was a Visiting Professor with the Electrical and Computer Engineering Department, Henry Samueli School of Engineering, University of California, Irvine, where he focused on antennas and systems miniaturization for wireless and sensing applications.

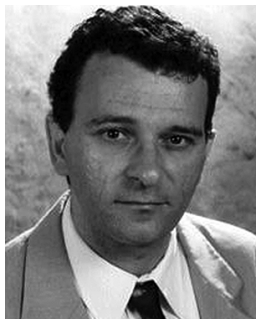

Franco De Flaviis was born in Teramo, Italy, in 1963. He received the laurea degree in electronics engineering from the University of Ancona, Italy, in 1990 and the M.S. and Ph.D. degrees in electrical engineering from the Department of Electrical Engineering, University of California, Los Angeles (UCLA), in 1994 and 1997, respectively.

In 1991, he was an Engineer at Alcatel as a Researcher specialized in the area of microwave mixer design. In 1992, he was a Visiting Researcher at UCLA working on low intermodulation mixers. Currently, he is an Associate Professor at the Department of Electrical and Computer Engineering, University of California, Irvine. His research interests are in the field of computer-aided electromagnetics for high-speed digital circuits and antennas and microelectromechanical systems for RF applications fabricated on unconventional substrates such as printed circuit board and microwave laminates. 